\title{
Clustering Evolving Networks
}

\author{
Tanja Hartmann, Andrea Kappes, Dorothea Wagner \\ Department of Informatics, Karlsruhe Institute of Technology (KIT) \\ \{tanja.hartmann, andrea.kappes, dorothea.wagner\}@kit.edu
}

\begin{abstract}
Roughly speaking, clustering evolving networks aims at detecting structurally dense subgroups in networks that evolve over time. This implies that the subgroups we seek for also evolve, which results in many additional tasks compared to clustering static networks. We discuss these additional tasks and difficulties resulting thereof and present an overview on current approaches to solve these problems. We focus on clustering approaches in online scenarios, i.e., approaches that incrementally use structural information from previous time steps in order to incorporate temporal smoothness or to achieve low running time. Moreover, we describe a collection of real world networks and generators for synthetic data that are often used for evaluation.
\end{abstract}

\section{Introduction}

Clustering is a powerful tool to examine the structure of various data. Since in many fields data often entails an inherent network structure or directly derives from physical or virtual networks, clustering techniques that explicitly build on the information given by links between entities recently received great attention. Moreover, many real world networks are continuously evolving, which makes it even more challenging to explore their structure. Examples for evolving networks include networks based on mobile communication data, scientific publication data, and data on human interaction.

The structure that is induced by the entities of a network together with the links between is often called graph, the entities are called vertices and the links are called edges. However, the terms graph and network are often used interchangeably. The structural feature that is classically addressed by graph clustering algorithms are subsets of vertices that are linked significantly stronger to each other than to vertices outside the subset. In the context of mobile communication networks this could be, for example, groups of cellphone users that call each other more frequently than others. Depending on the application and the type of the underlying network, searching for this kind of subsets has many different names. Sociologists usually speak about community detection or community mining in social networks, in the context of communication networks like Twitter, people aim at detecting emerging topics while in citations networks the focus is on the identification of research areas, to name but a few. All these issues can be solved by modeling the data as an appropriate graph and applying graph clustering. The found sets (corresponding to communities, topics or research areas) are then called clusters and the set of clusters is called a clustering. We further remark that also beyond sociology the term community is often used instead of cluster [102]. The notion of clusters or communities as densely connected subgroups that are only sparsely connected 
to each other has led to the paradigm of intracluster density versus intercluster sparsity in the field of graph clustering.

Nevertheless, the notion of a clustering given so far still leaves room for many different formal definitions. Most commonly, a clustering of a graph is defined as a partition of the vertex set into subsets, which form the clusters. In some scenarios (e.g., outlier detection) it is, however, undesirable that each vertex is assigned to a cluster. In this case, a clustering not necessarily forms a partition of the vertex set, but leaves some vertices unclustered. Yet both concepts are based on disjoint vertex sets, and the latter can be easily transformed into the former one by just considering each vertex that is not a member of a cluster as a cluster consisting of exactly one vertex. Other applications further admit overlapping clusters, again with or without a complete assignment of the vertices to clusters.

In this survey we give an overview of recent graph clustering approaches that aim at finding disjoint or overlapping clusters in evolving graphs. The evolution of the graph is usually modeled following one of two common concepts: The first concept is based on a series of snapshots of the graph, where each snapshot corresponds to a time step, and the difference between two consecutive snapshots results from a bunch of edge and vertex changes. The second concept considers a given stream of atomic edge and vertex changes, where each change induces a new snapshot and a new time step. The primary objective of clustering such networks is to find a meaningful clustering for each snapshot. Some algorithms further aim at a particularly fast computation of these clusterings, others assume that changes have only a small impact on the community structure in each time step, and thus, aim at clusterings that differ not too much in consecutive time steps. The latter was introduced as temporal smoothness by Chakrabarti et al. [31] in the context of clustering evolving attributed data (instead of graphs). In order to achieve these goals, online algorithms explicitly exploit information about the graph structure and the community structure of previous time steps. Algorithms that further use structural information from following time steps are called offline. In this survey, we consider only online algorithms that can be roughly separated into two classes. The first class contains clustering approaches that incorporate temporal smoothness inspired by Chakrabarti et al. Most of these approaches are based on an existing static clustering algorithm, which is executed from scratch in each time step (cp. Figure 1). In contrast, the approaches in the second class dynamically update clusterings found in previous time steps without a computation from scratch (cp. Figure 2).

Apart from finding an appropriate clustering in each snapshot of an evolving graph, many applications require further steps in order to make the found clusterings interpretable and usable for further analysis. A first natural question directly resulting from the evolution of the graph is how the found clusters or communities evolve over time and at what time steps events like cluster merging or cluster splitting occur. In order to answer this question, the clusters need to be tracked over time, thereby finding sequences of snapshots where certain clusters remain stable while other clusters may split or merge. In this context, clusters or communities of a single snapshot are often called local in order to distinguish them from sequences of associated (local) communities in consecutive snapshots, which describe the evolution of a certain (meta)community over time. When the evolution of the clusters is supposed to be interpreted by human 


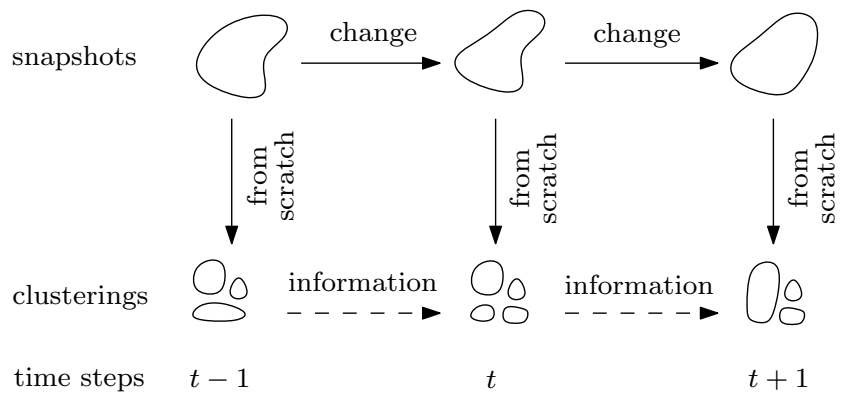

Fig. 1: Evolutionary clustering strategy in evolving graphs. Horizontal dashed arrows indicate the use of information, vertical arrows indicate the evolutionary strategy based on a static clustering approach applied from scratch.

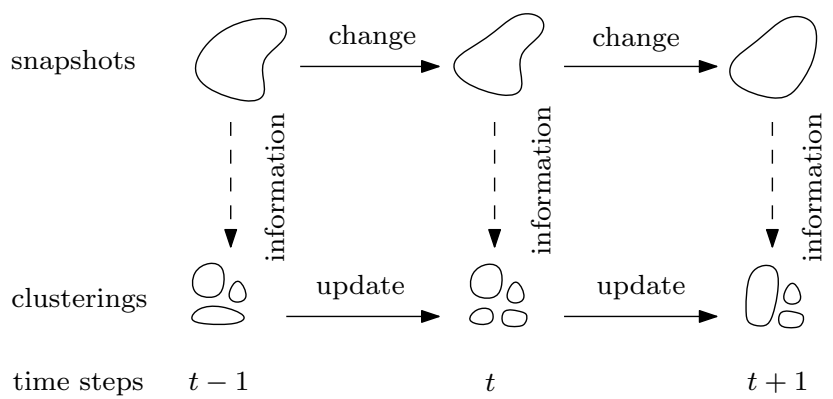

Fig. 2: Dynamic update strategy for clusterings in evolving graphs. Vertical dashed arrows indicate the use of information, horizontal arrows indicate the dynamic update strategy based on previous time steps.

experts, it is further necessary to present the algorithmic results in a clear and readable form. Hence, the visualization of evolving clusters is another central issue in the context of clustering evolving graphs. The evaluation of found clusterings is finally an issue regarding the design of good clustering algorithms. There are many open questions on how to choose an appropriate evaluation scheme in order to get credible and comparable results. We discuss these issues in more detail in Section 1.1 and give a brief idea on applications based on clustering evolving graphs in Section 1.3.

Delimitation. Apart from clustering approaches that follow the intracluster density versus intercluster sparsity paradigm, there exist various further approaches that look very similar at a first glance but turn out to have a different focus.

Very closely related to graph clustering are algorithms for graph partitioning [19]. In contrast to many graph clustering algorithms, graph partitioning always assumes that the number of clusters is an input parameter, most often, a power of 2, and seeks to minimize the number of edges cut by a partition, such that the parts have (almost) equal size. Its main application is not network analysis but the preprocessing of graphs for parallel computing tasks. The dynamic counterpart to static graph partitioning is often 
called repartitioning or load balancing $[28,38,93]$. Another area similar to clustering evolving graphs is clustering graph streams [3,159]. Similar to consecutive graph snapshots, a graph stream is a sequence of consecutively arriving graphs, but instead of finding a clustering of the vertices in each graph or snapshot, the aim is to detect groups of similar graphs. The term streaming algorithm is usually used for algorithms that process the data in one or few passes under the restriction of limited memory availability, like for example the partitioning algorithm by Stanton and Kliot [135]. However, some authors also use the adjective streaming or the term stream model in the context of graph changes in order to describe consecutive atomic changes $[8,48]$. A further task is the search for stable subgraphs in a given time interval in an evolving network, i.e., subgraphs that change only slightly during the whole interval. Depending on the formal definition of stability these subgraphs have various other names, like heavy subgraphs or high-score subgraphs [22]. Pattern Mining in evolving graphs is focused on frequently occurring subgraphs, independent from their density [23].

Intention and Outline. In this survey, we introduce some of the current graph clustering approaches for evolving networks that operate in an online scenario. All approaches have in common that they use structural information from the previous time steps in order to generate a meaningful clustering for the snapshot of the current time step. In doing so, some approaches focus on temporal smoothness, while other approaches aim at a fast running time and a few even achieve both.

In contrast to existing surveys on graph clustering, we focus on online algorithms in evolving networks. A very detailed and well-founded presentation of algorithmic aspects in static (non-evolving) and evolving graphs is further given in the theses of Görke [65]. For an overview on clustering techniques in static graphs see also Schaeffer [127] and Fortunato et al. [57]. The latter also provide a short abstract on clustering evolving graphs. Aynaud et al. [11] explicitly consider clustering approaches in evolving graphs, however, they do not focus on the algorithmic aspect of reusing structural information in an online scenario. Finally, Bilgin and Yener [20] consider evolving networks from a more general perspective. They also provide a section on "Clustering Dynamic Graphs", however, the emphasis of this section is the above mentioned idea of clustering graph streams.

This paper is organized as follows. In Section 1.1, we discuss the above mentioned main issues related to clustering evolving networks in more detail. In Section 1.2 we provide an overview on popular quality and distance measures for clusterings. The former are of course used for the evaluation of clusterings but also in the context of algorithm design. The latter are used for the evaluation as well as for cluster tracking and event detection. We conclude the introduction by a a brief idea on applications in Section 1.3. The main part of this survey is presented in Section 2, where we introduce current clustering approaches according to our focus described above. Moreover, we provide an overview on the main features of the presented algorithms in Table 1. Section 3 further lists a selection of data sets and graph generators used for the evaluation of the approaches presented in Section 2 and briefly discusses the difficulties in choosing appropriate data for evaluating clustering approaches on evolving graphs. We finally conclude in Section 4. 
Notation. Until noted otherwise, we will assume that graphs are simple, i.e., they do not contain loops and parallel edges. A dynamic or evolving graph $\mathcal{G}=\left(G_{0}, \ldots, G_{t_{\max }}\right)$ is a sequence of graphs with $G_{t}=\left(V_{t}, E_{t}\right)$ being the state of the dynamic graph at time step t. $G_{t}$ is also called snapshot of $\mathcal{G}$. A clustering $\mathcal{C}_{t}=\left\{C_{1}, \ldots, C_{k}\right\}$ of $G_{t}$ is a set of subsets of $V_{t}$ called clusters or communities. If these subsets are pairwise disjoint, the clustering is called disjoint, otherwise it is overlapping. A disjoint clustering that further has the property that each vertex is contained in a cluster, i.e., that corresponds to a partition of $V_{t}$, is called complete. Complete clusterings are often represented by storing a cluster id for each vertex that encodes the corresponding cluster. A pair $\{u, v\}$ of vertices such that there is a cluster that contains both $u$ and $v$ is called intracluster pair, otherwise $\{u, v\}$ is called intercluster pair. An edge between the vertices of an intracluster pair is called intracluster edge; intercluster edges are defined analogously. A singleton clustering is a complete clustering where each cluster contains only one vertex; such clusters are called singleton clusters. The other extreme, i.e., a clustering consisting of only one cluster containing all vertices, is called 1-clustering. Each cluster $C \subset V_{t}$ further induces a cut in $G_{t}$. A cut in a graph $G=(V, E)$ is defined by a set $S \subset V$, which indicates one side of the cut. The other side is implicitly given by $V \backslash S$. A cut is thus denoted by $(S, V \backslash S)$.

\subsection{Main Issues When Clustering Evolving Networks}

In the following we briefly discuss the main issues related to clustering evolving networks. We consider cluster tracking and visualization first, since these problems can be solved independent from the cluster detection. Our remarks on cluster detection in online scenarios give a rough idea on different techniques used in this field, followed by a short overview on some state-of-the-art evaluation techniques.

Cluster Tracking and Event Detection. Assuming the cluster structure of the network is already given for each snapshot by an arbitrary clustering approach, detecting the evolution of the clusters over time becomes a task independent from finding the clusters. Most approaches that address this task describe a framework of two subproblems. On the one hand, they seek for series of similar clusters in consecutive snapshots (often called meta communities, meta groups or time-lines), and on the other hand, they aim at identifying critical events where clusters, for instance, appear, survive, disappear, split or merge. In particular, deciding if a cluster that has just disappeared reappears in future time steps, and thus, actually survives, requires future information, which is not available in an online scenario. Hence, whether a framework is applicable in an online scenario depends on the defined events. The frameworks of Takaffoli et al. [141] and Green et al. [73] are offline frameworks since they compare the structure of the clusters of the current snapshot to all previous and future snapshots in order to also find clusters that disappear and reappear after a while. While the framework by Takaffoli et al. requires disjoint clusters, the approach of Green et al. also allows overlapping clusters.

In order to compare clusters of consecutive snapshots, many approaches define a similarity measure considering two clusters as similar if the similarity value exceeds a given threshold. Asur et al. [10] detect similar clusters by the size of their intersection. 
Takaffoli et al. [141] use the size of the intersection over the size of the larger cluster. Berger-Wolf and Saia [18] admit the use of any similarity measure that is efficiently computable and satisfies some mathematical properties, as it does the standard Jaccard similarity measure [77] that describes the size of the intersection over the size of the union.

The frameworks mentioned so far can be applied to any cluster structure in a given graph, regardless which clustering approach was used to find this structure. Other tracking approaches, however, exploit special properties of the given cluster structures in the snapshots, and thus, require that the clusters are constructed by a designated (static) clustering method. Palla et al. [109] require clusterings found by the clique percolation method (PCM) [41], which can be also seen as a special case of a clustering method proposed by Everett and Borgatti [51]. For a brief description of PCM see the part in Section 2 where algorithms maintaining auxiliary structures are introduced. In order to identify evolving clusters in two consecutive time steps, Palla et al. construct the union of the two corresponding snapshots and apply again PCM to this union graph. Due to the properties of the clusters found by PCM, it holds that each cluster found in one of the snapshots is contained in exactly one cluster in the union graph. A cluster $C$ in the snapshot at time $t-1$ is then associated with the cluster $C^{\prime}$ in the snapshot at time $t$ that is contained in the same cluster in the union graph and has the most vertices in common with $C$. Falkowski et al. [53] consider clusters that result from a hierarchical divisive edge betweenness clustering algorithm. In contrast to Palla et al. who map clusters only between two consecutive time steps, Falkowski et al. present an offline approach. They construct an auxiliary graph that consists of all clusters found in any snapshot and two clusters are connected by an edge if and only if the relative overlap of both clusters exceeds a given threshold. On this graph the authors apply the same clustering algorithm as on the snapshots in order to find groups of (local) communities that are similar in different time steps. Obviously, this approach is an offline approach. Another offline approach is given by Tantipathananandh et al. [17]. Here the authors assume given groups in the snapshots, where members of the same group interact while members of different groups do not interact. Based on observed interactions of entities in consecutive time steps, an auxiliary graph is built on which the authors solve a coloring problem. Finally, there are also some frameworks that are not especially designed for networks, but are general enough to be applied to networks as well [134]. For a further categorization of tracking methods see also Aynaud et al. [11].

Besides whole frameworks that track clusters according to similarity, tracking clusters by cluster ids is a very natural and simple approach. This however requires that clusters that are given the same id in two consecutive time steps are somehow related. Many graph clustering algorithms that dynamically update previous clusterings, like LABEL PROPAGATION [111], LABELRANKT [151] and DiDiC [60], to name but a few of the approaches introduced in Section 2, fulfill this requirement. The relation between clusters of the same id depends on how the particular algorithm chooses the cluster ids. Furthermore, inferring clusters based on generative models as done by FACETNET [91] often admits the tracking of clusters without an additional framework, as the evolution of the clusters can be read off the resulting model. 
Visualization of the Evolution of Clusters. Even if there is already a clustering given for each snapshot and we know which local clusters correspond to each other in different snapshots, the visualization of the evolution of these clusters is still not trivial. Since the evolution of clusters involves not only one graph but several snapshots of an evolving graph, the total object that needs to be visualized quickly becomes very complex. Hence, one problem that needs to be dealt with when visualizing such an object is simplification. Many visualization approaches and in particular interactive visualization tools solve this problem by offering different views on different aspects of the object $[32,53,125]$. Apart from different views, the most intuitive layout for evolving clusterings is probably to draw consecutive snapshots next to each other and depict the correspondence of clusters for example by colors or by additional edges between the snapshots. In this kind of layout another popular task in the field of visualization gains importance, namely the preservation of the mental map. This means that corresponding clusters (and also vertices) in different snapshots are placed at similar positions in the image of the particular snapshot, such that potential changes in the clustering structure can be easily recognized. The goal to generate well readable diagrams further also justifies the postulation of temporal smoothness. Instead of drawing the snapshots next to each other, TeCFlow [63] and SoNIA [97] provide the possibility to create little movies out of consecutive snapshots. Moreover, many visualization approaches are proposed on top of new clustering approaches or existing tracking frameworks [75]. However, these approaches are often specially tailored with respect to these clustering algorithms or tracking frameworks.

Online Cluster Detection in Evolving Graphs. The most intuitive attempt to deal with clusters in evolving graphs is to cluster each snapshot independently with a static clustering algorithm and track the clusters afterwards in order to uncover their evolution. However, depending on the static clustering algorithm that is used to find the clusters, the structure of the clusters in each snapshot may vary greatly such that a tracking method may find a lot of change points instead of nicely evolving clusters. Greedy agglomerative approaches, for example, that aim at optimizing an objective function often tend to find different local optima in consecutive snapshots, depending on the order in which the vertices are chosen. Hopcroft et al. [76] were some of the first authors who clustered snapshots with the help of a static clustering algorithm and then tracked the found clusters over time. They overcome the problem that even small perturbations in the underlying graph may lead to significant changes in the structure of the found clusters in consecutive snapshots by applying a greedy agglomerative approach several times with different orderings of vertices. Only clusters that remain stable under such multiple clustering runs (so called natural or consensus clusters) are then considered for the tracking.

Another way to overcome the problem of unstable clusterings is to explicitly incorporate temporal smoothness in the clustering process. A first attempt in this direction was done by Chakrabarti et al. [31] in 2006; however, they clustered attributed data instead of graphs. Their idea is to exploit the knowledge about the previously found clustering to find a clustering for the current time step that is similar to the previous clustering (i.e., has low history cost) and is still a good clustering also for the data in the current time step (i.e., has high snapshot quality). Depending on the clustering algo- 
rithm in which the temporal smoothness is incorporated, this may lead to an objective function that needs to be optimized or to an adaption of the input data or certain parameters. Chakrabarti et al. examine two widely used clustering algorithms within their framework; k-means and agglomerative hierarchical clustering. Their technique of incorporating temporal smoothness into static clustering approaches has been established under the name evolutionary clustering. It has been adapted to graph clustering by Kim and Han [80], Chi et al. [34], Görke et al. [69] and Xu et al. [153]. The FACETNET approach by Lin et al. [91] is based on a generic model that is equivalent to the framework of Chakrabarti et al. under certain assumptions. The corresponding algorithms are described in more detail in Section 2. Note that the term evolutionary is not limited to evolutionary clustering (as introduced by Chakrabarti et al.). It is also used in many other contexts, like, for example, in the context of evolutionary search heuristics.

Under the term dynamic graph clustering we subsume the remaining clustering approaches presented in this survey. The difference to evolutionary clustering, where in each time step a static algorithm is applied from scratch, is that dynamic approaches update existing information from previous time steps without recalculating the whole clustering from scratch. This can be done, for example, by reusing parts of the previous clustering and just updating local areas where the clustering has become infeasible [1] or, in case of greedy agglomerative algorithms, initializing the current clustering with the previously found clusters [142]. Other approaches update auxiliary information like sets of dense subsets [8], lists of well connected neighbors [82], eigenvectors [106] or structures like cut trees [67] or clique graphs [48]. Quite a few approaches also combine these techniques. Although most of these dynamic approaches aim at reducing the running time, in many cases updating previous information implicitly also leads to temporal smoothness. Other update methods admit the detection of cluster events like splitting or merging $[54,67]$.

Evaluation of Clustering Methods. As the (rather fuzzy) definition of graph clustering according to the intracluster density versus intercluster sparsity paradigm does not correspond to a well defined optimization problem, the comparison of different clustering approaches is inherently difficult. One aspect that should always be taken into account is scalability, i.e., to what extent the running time increases with the graph size. Disregarding the influence of more or less efficient implementations and hardware environments, the scalability of algorithms can be easily compared and evaluated. In some cases, it is possible to consider the immediate usefulness of a clustering for a certain application, which allows to compare different clusterings in a precise and well motivated way. An example for this is the use of dynamic graph clustering in the context of routing protocols in Mobile Ad hoc Networks (MANETS), where clusterings can be evaluated based on their impact on statistics as Delivery Ratio or Average Delivery Time [46, 104]. However, most applications do not yield such statistics, which is why most authors focus on two main approaches to evaluate clusterings, both of which are not undisputed.

The first one is the evaluation of clusterings with the help of a ground truth clustering. For real world data, in most cases this corresponds to additional metadata that indicate well motivated communities. In the context of synthetic data this usually refers to clusters implanted in the graph structure during generation. Usually, a ground truth 
clustering either corresponds to a partition of the objects in the context of algorithms finding complete clusterings, or a set of subsets of objects in the context of algorithms admitting overlapping clusters. Ground truth clusterings can now be compared to the outcome of an algorithm with the help of a suitable distance measure on partitions or sets of objects. In the next section we introduce some distance measures that can be used to evaluate clusterings based on ground truth clusterings. Moreover, Section 3 gives an overview of real world datasets used in the literature both with and without metadata, and describes some models that can be used to generate synthetic data. In case the metadata about real world data is not available in form of a ground truth clustering, it is further possible to manually look into the data and perform plausibility checks of the clusterings obtained. However, this approach requires a lot of interpretation and, due to the large size of some datasets, is often necessarily limited to a subset of the data at hand.

The second main approach is the use of quality measures to evaluate the goodness of a given clustering. Using quality measures simplifies the evaluation a lot, as it turns the inherently vague definition of clustering into an explicit optimization problem. Some algorithms use these objective functions explicitly and optimize them by using for example local search techniques or trying to find provably optimal solutions efficiently $[15,43,44,46,69,104,118]$. Others use objective functions as an additional evaluation criterion, although the algorithm itself does not explicitly optimize any measure [60,151]; often, the authors then do not claim to obtain the best values according to the considered measure, but use the results as an additional sanity check to motivate their approach, together with experiments involving ground truth clusterings. We will give the definitions of some commonly used quality measures in the next section.

For a further discussion on the difficulties of evaluating community detection methods and a brief history of method evaluation, see [86].

\subsection{Quality and Distance Measures for Clusterings}

In this section, we will give a short overview of quality measures assessing the goodness of clusterings, followed by a discussion on what has to be additionally considered in the dynamic scenario, and an introduction to some frequently used distance measures that can be used to evaluate the similarity of two clusterings. To give a comprehensive overview of all quality and distance measures used in the literature is beyond the scope of this article; further information can be found for example in the articles of Fortunato [57] and Wagner et al. [147].

Quality Measures in Static Graphs. We will describe two main approaches to measure the quality of a clustering in the static scenario, the first one is based on balanced cuts and the second on null models. For better readability, we consider only unweighted graphs in this section. Note that all measures described here can be generalized to weighted graphs in a straightforward way.

The use of cuts as a means to analyze community structures in networks has a long tradition [158]. A trivial community detection algorithm could for example determine the minimum cut in a graph, split the graph according to this cut, and recurse this procedure on the resulting communities until some termination criterion, as for example 
a desired number of clusters, is met. This procedure at least guarantees that two found communities or clusters are locally not too strongly connected by intercluster edges. Nevertheless, this often leads to clusters of very uneven sizes; especially in the presence of low degree vertices, minimum cuts tend to separate only one or few vertices from the remainder of the graph. For this reason, clustering algorithms typically build upon balanced cuts, i.e., cuts that simultaneously cross few edges and split the graph in two approximately equal sized parts.

Probably the first formal definitions of balanced cuts used in the context of graph clustering are the measure conductance and expansion [78]. For a subset $S$, let $e(S, V \backslash$ $S$ ) denote the number of edges linking vertices in $S$ with vertices in $V \backslash S$. Furthermore, the volume $\operatorname{vol}(S):=\sum_{v \in S} \operatorname{deg}(v)$ of a subset of vertices $S$ is defined as the sum of the degrees of its vertices. Then, the conductance cond of a cut $(S, V \backslash S)$ can be written as:

$$
\operatorname{cond}(S, V \backslash S)=\frac{e(S, V \backslash S)}{\min \{\operatorname{vol}(S), \operatorname{vol}(V \backslash S)\}}
$$

Many variations thereof exist, most of which either replace the volume by other notions of cluster size, or use a slightly different tradeoff between the cut size and the sizes of the two induced parts. We give the definition of two of these variation, namely expansion and normalized cut:

$$
\begin{aligned}
\exp (S, V \backslash S) & =\frac{e(S, V \backslash S)}{\min \{|S|,|V \backslash S|\}} \\
\operatorname{ncut}(S, V \backslash S) & =\frac{e(S, V \backslash S)}{\operatorname{vol}(S)}+\frac{e(S, V \backslash S)}{\operatorname{vol}(V \backslash S)}
\end{aligned}
$$

The latter definition is especially popular in the field of image segmentation [130]. Finding an optimal cut with respect to any of the three definitions above is $\mathcal{N} \mathcal{P}$-hard [88, $130,132]$, which is why divisive algorithms are usually based on approximation algorithms or heuristics. It remains to mention that cut-based measures are closely related to spectral clustering techniques [146].

It is not immediately clear how the above measures can be used to evaluate whole clusterings. One possibility is to associate two values with each cluster $C$, one that evaluates the cut that separates the cluster from the rest of the graph and another evaluating all cuts within the subgraph that is induced by $C$. In the context of conductance, this leads to the following definition of inter- and intracluster conductance of a cluster $C$ [26]:

$$
\begin{aligned}
& \text { intercluster conductance }(C)=\operatorname{cond}(C, V \backslash C) \\
& \text { intracluster conductance }(C)=\min _{S \subset C}\{\operatorname{cond}(S, C \backslash S)\}
\end{aligned}
$$

In a good clustering according to the intracluster density versus intercluster sparsity paradigm, the intracluster conductance of the clusters is supposed to be high while their intercluster conductance should be low. An overall value for the intracluster conductance of a whole clustering can then be obtained by taking, for example, the minimum or average of the intracluster conductance over all clusters [70]. Analogously, the intercluster conductance of a clustering can be defined as the maximum intercluster conductance over all clusters. This leads to a bicriterial optimization problem. Calculating the 
intracluster conductance of a cluster is $\mathcal{N} \mathcal{P}$-hard, which immediately follows from the $\mathcal{N} \mathcal{P}$-hardness of finding a cut with optimum conductance. The same holds if we replace conductance by expansion or normalized cut. Hence, most clustering approaches that aim at solving the resulting optimization problem are again based on approximation algorithms or heuristics. The cut clustering algorithm of Flake et al. [56, 67], however, guarantees at least a lower bound of the intracluster expansion of the found clusters. In principal, these cut based criteria can be also used to evaluate overlapping clusterings, although they are much more common in the context of complete clusterings.

Another approach to measure the goodness of clusterings that has gained a lot of attention during the last decade is the use of null models. Roughly speaking, the idea behind this is to compare the number of edges within clusters to the expected number of edges in the same partition, if edges are randomly rewired. The most popular measure in this context is the modularity of a clustering as defined by Girvan and Newman [103] in 2004. Let $e(C)$ denote the number of edges between the vertices in cluster $C$. Then, the modularity $\bmod (\mathcal{C})$ of a (complete) clustering $\mathcal{C}$ can be defined as

$$
\bmod (\mathcal{C})=\sum_{C \in \mathcal{C}} \frac{e(C)}{m}-\sum_{C \in \mathcal{C}} \frac{\operatorname{vol}(C)^{2}}{4 m^{2}} .
$$

Here, the first term measures the actual fraction of edges within clusters and the second the expectation of this value after random rewiring, given that the probability that a rewired edge is incident to a particular vertex is proportional to the degree of this vertex in the original graph. The larger the difference between these terms, the better the clustering is adjusted to the graph structure. The corresponding optimization problem is $\mathcal{N} \mathcal{P}$-hard [24]. Modularity can be generalized to weighted [101] and directed $[9,87]$ graphs, to overlapping or fuzzy clusterings $[105,129]$, and to a local scenario, where the goal is to evaluate single clusters $[33,35,92]$. Part of its popularity stems from the existence of heuristic algorithms that optimize modularity and that are able to cluster very large graphs in short time $[21,108,121]$. In Section 2, we will describe some generalizations of these algorithms to the dynamic setting. Furthermore, in contrast to many other measures and definitions, modularity does not depend on any parameters. This might explain why it is still widely used, despite some recent criticism [58].

Quality Measures in Evolving Graphs. In the context of dynamic graph clustering, we aim at clusterings of high quality for each snapshot graph. Compared to the static approach, as discussed in Section 1, temporal smoothness becomes an additional dimension. Speaking in terms of objective functions, we would like to simultaneously optimize the two criteria quality and temporal smoothness.

As already mentioned before, one approach to obtain this is introduced by Chakrabarti et al. [31]. The idea is to measure the snapshot quality sq of the current clustering $\mathcal{C}_{t}$ at time step $t$ (with respect to the current snapshot $\mathcal{G}_{t}$ ) by a (static) measure for the goodness of a clustering. Similarly, the smoothness is measured by the history cost hc of the current clustering, which is usually defined as the distance of the current clustering to the previous clustering $\mathcal{C}_{t-1}$ at time step $t-1$. The snapshot quality could for example be measured by modularity and the smoothness by any of the distance measures introduced in the next paragraph. The goal is then to optimize a 
linear combination of both measures, where $\alpha$ is an input parameter that determines the tradeoff between quality and smoothness:

$$
\operatorname{minimize} \alpha \cdot \operatorname{sq}\left(\mathcal{C}_{t}, \mathcal{G}_{t}\right)-(1-\alpha) \cdot \operatorname{hc}\left(\mathcal{C}_{t}, \mathcal{C}_{t-1}\right) .
$$

Closely related to this approach, but not relying on an explicit distance measure, is the claim that a good clustering of the snapshot at time step $t$ should also be a good clustering for the snapshot at time step $t-1$. This is based on the underlying assumption that fundamental structural changes are rare. Hence, linearly combining the snapshot quality of the current clustering with respect to the current snapshot $\mathcal{G}_{t}$ and the previous snapshot $\mathcal{G}_{t-1}$ yields a dynamic quality measure, which can be build from any static quality measure:

$$
\text { minimize } \alpha \cdot \operatorname{sq}\left(\mathcal{C}_{t}, \mathcal{G}_{t}\right)+(1-\alpha) \operatorname{sq}\left(\mathcal{C}_{t}, \mathcal{G}_{t-1}\right) .
$$

This causes the clustering at time step $t$ to also take the structure of snapshot $\mathcal{G}_{t-1}$ into account, which implicitly enforces smoothness. Takaffoli et al. [142] apply this approach in the context of modularity, and Chi et al. [34] in the context of spectral clustering; both will be discussed in Section 2 .

Distance Measures for Clusterings. In the context of graph clustering, distance measures have three main applications. First, similar to static clustering, they can be used to measure the similarity to a given ground truth clustering. Second, they can be used as a measure of smoothness, for example by comparing the clusterings of adjacent time steps. Third, they are useful in the context of event detection; a large distance between two consecutive clusterings may indicate an event. A plethora of different measures exist in the literature, none of which is universally accepted. For this reason, we will only introduce the measures used by the dynamic algorithms we describe in Section 2. This includes the probably best known index in the context of clustering, the normalized mutual information. If not mentioned otherwise, all clusterings considered in this section are assumed to be complete.

Mutual information has its roots in information theory and is based on the notion of the entropy of a clustering $\mathcal{C}$. For a cluster $C \in \mathcal{C}$, let $P(C):=|C| / n$. With that, the entropy $\mathcal{H}$ of $\mathcal{C}$ can be defined as

$$
\mathcal{H}(\mathcal{C}):=-\sum_{C \in \mathcal{C}} P(C) \log _{2} P(C)
$$

Similarly, given a second clustering $\mathcal{D}$, with $P(C, D):=|C \cap D| / n$, the conditional entropy $H(\mathcal{C} \mid \mathcal{D})$ is defined as

$$
\mathcal{H}(\mathcal{C} \mid \mathcal{D}):=\sum_{C \in \mathcal{C}} \sum_{D \in \mathcal{D}} P(C, D) \log _{2} \frac{P(C)}{P(C, D)}
$$

Now the mutual information $\mathcal{I}$ of $\mathcal{C}$ and $\mathcal{D}$ can be defined as

$\mathcal{I}(\mathcal{C}, \mathcal{D}):=\mathcal{H}(\mathcal{C})-\mathcal{H}(\mathcal{C} \mid \mathcal{D})=\mathcal{H}(\mathcal{D})-\mathcal{H}(\mathcal{D} \mid \mathcal{C})=\sum_{C \in \mathcal{C}} \sum_{D \in \mathcal{D}} P(C, D) \log _{2} \frac{P(C, D)}{P(C) P(D)}$ 
Informally, this is a measure of how much information the knowledge that a vertex belongs to a certain cluster in clustering $\mathcal{D}$ yields about its cluster id in $\mathcal{C}$. Several normalizations of this measure exist; according to Fortunato [57], the most commonly used normalization is the following notion of normalized mutual information (NMI), which maps the mutual information to the interval $[0,1]$ :

$$
\operatorname{NMI}(\mathcal{C}, \mathcal{D})=\frac{2 \mathcal{I}(\mathcal{C}, \mathcal{D})}{\mathcal{H}(\mathcal{C})+\mathcal{H}(\mathcal{D})}
$$

Technically, this is not a distance but a similarity measure, as high values of NMI indicate high correlation between the clustering. If need be, it can be easily turned into a distance measure by considering $1-\operatorname{NMI}(\mathcal{C}, \mathcal{D})$. There also exists a generalization to overlapping clusterings [84]. Among the approaches we describe in Section 2, Yang et al. [155], Cazabet et al. [29] and Kim and Han [80] use mutual information to compare against ground truth clusterings. In contrast to that, Lin et al. [91] use it to compare the time step clusterings to the communities of the aggregated graph, which can be seen as both a measure of smoothness and comparison to some kind of ground truth clustering. Wang et al. [82] use NMI both to measure the similarity of a clustering to a generated ground truth clustering and to compare the results of an approximation algorithm to clusterings found by an exact algorithm (according to their definition of clusters).

Aynaud and Guillaume [12] use, as an alternative to NMI, the minimum number of vertex moves necessary to convert one clustering into the other as a measure of distance. Their main argument to consider this approach is that absolute values are far easier to interpret.

Another very intuitive measure for the distance between two partitions is the Rand index introduced by Rand [117] in 1971. Let $s(\mathcal{C}, \mathcal{D})$ be the number of vertex pairs that share a cluster both in $\mathcal{C}$ and $\mathcal{D}$ and $d(\mathcal{C}, \mathcal{D})$ the number of vertex pairs that are in different clusters both in $\mathcal{C}$ and $\mathcal{D}$. With that, the Rand index $\mathcal{R}$ of $\mathcal{C}$ and $\mathcal{D}$ is defined as

$$
\mathcal{R}(\mathcal{C}, \mathcal{D}):=1-\frac{s(\mathcal{C}, \mathcal{D})+d(\mathcal{C}, \mathcal{D})}{\left(\begin{array}{l}
n \\
2
\end{array}\right)}
$$

This corresponds to counting the number of vertex pairs where both clusterings disagree in their classification as intracluster or intercluster pair, followed by a normalization. Delling et al. [40] argue that this measure is not appropriate in the context of graph clustering, as it does not consider the topology of the underlying graph. They propose to only consider vertex pairs connected by an edge, which leads to the graph based Rand index. This graph based version is used by Görke et al [69] to measure the distance between clusterings at adjacent time steps.

Chi et al. [34] use the chi square statistic to enforce and measure the similarity between adjacent clusterings. The chi square statistic was suggested by Pearson [114] in 1900 to test for independence in a bivariate distribution. In the context of comparing partitions, different variants exist [96]; the version used by Chi et al. is the following:

$$
\chi^{2}(\mathcal{C}, \mathcal{D})=n \cdot\left(\sum_{C \in \mathcal{C}} \sum_{D \in \mathcal{D}} \frac{|C \cap D|}{|C| \cdot|D|}-1\right)
$$




\subsection{Applications}

Graph clustering has possible applications in many different disciplines, including biology and sociology. Biologists are for example interested in how diseases spread over different communities, sociologists often focus on cultural and information transmission. Many of the networks analyzed in these areas have a temporal dimension that is often neglected; taking it into account potentially increases the usefulness of clustering for the respective application and at the same time evokes new challenges like for example the involvement of temporal smoothness. In the context of social networks, the benefit of temporal smoothness becomes in particular obvious, since social relations and resulting community structures are not expected to change frequently. Giving an exhaustive list of application areas is beyond the scope of this article; some further information can be found in the overview article of Fortunato [57]. Instead, we will give some examples where clustering approaches designed for evolving graphs have clearly motivated advantages over static approaches.

A little-known but very interesting application of graph clustering is the use in graph drawing or visualization algorithms. The general idea is to first cluster the vertices of the graph and then use this information in the layouting steps by placing vertices in the same community in proximity of each other. This has several advantages: The layout makes the community structure of the graph visible, which is desirable in many applications. Furthermore, he intracluster density versus intracluster sparsity paradigm causes many edges to be within clusters, which in turn corresponds to small edge lengths. Last but not least, layout algorithms that use clustering as a preprocessing step are usually quite fast. As an example, Muelder and Ma have used clustering algorithms in combination with layouts based on treemaps [98] and space filling curves [99]. A straightforward extension to these approaches is the task to visualize dynamic graphs [125]. Dynamic clustering algorithms can help in this context to reduce the running time for the preprocessing in each time step. Furthermore, if they are additionally targeted at producing smooth clusterings, this results in smoother layouts, or, in terms of layout algorithms, in a good preservation of the mental map.

Another interesting application of dynamic graph clustering is its use in routing protocols in Mobile Ad hoc Networks (MANETS). On-Demand forwarding schemes for this problem discover paths in the network only when receiving concrete message delivery requests. It has been shown that "routing strategies based on the discovery of modular structure have provided significant performance enhancement compared to traditional schemes". [46] Due to the mobility of actors in the network, the resulting topology is inherently dynamic; recomputing the clustering whenever a change occurs is costly and requires global information. This motivated a number of online algorithms for modularity based dynamic clustering algorithms, with experiments showing that the use of the dynamic clustering improved the performance of forwarding schemes in this scenario [46, 104]. Another interesting aspect of this application is that "consistent modular structures with minimum changes in the routing tables" [46] are desirable, again motivating temporal smoothness. 


\section{Online Graph Clustering Approaches}

In this section we introduce current clustering algorithms and community detection approaches for evolving graphs. As discussed above, we consider only algorithms that operate in an online scenario, i.e., that do not use information from future time steps, and are incremental in the sense that they incorporate historic information from previous time steps to achieve temporal smoothness or a better running time. We use different categories to classify the approaches presented here. Some categories are associated with particular algorithmic techniques, other categories with applications or the form of the resulting clusterings. Apart from these categories, the GRAPHSCOPE approach [137] is presented at the beginning of this section, as it is one of the first and most cited dynamic approaches. The section concludes with two further approaches, which do not fit into one of the previous categories.

GraphScope. The GRAPHSCOPE approach by Sun et al. [137] is one of the first and most cited dynamic clustering approaches so far. However, contrary to the notion of communities as densely connected subgraphs, GRAPHSCOPE follows the idea of block modeling, which is another common technique in sociology. The aim is to group actors in social networks by their role, i.e., structural equivalence. Two actors are equivalent if they interact in the same way with the same actors (not necessarily with each other). This is, the subgraph induced by such a group may be disconnected or even consisting of an independent set of vertices. The latter is the case in approaches like GRAPHSCOPE that consider bipartite graphs of source and destination vertices and seek for groups of equivalent vertices in each part, i.e., groups consisting either of source or destination vertices. Furthermore, instead of independent snapshots, GRAPHSCOPE considers whole graph segments, which are sequences of similar consecutive snapshots that (w.l.o.g.) have all the same number of sources and destinations. The main idea is the following. Given a graph segment and a partition of the vertices in each part (the same partition for all snapshots in the graph segment), the more similar the vertices are per group the cheaper are the encoding costs for the graph segment using an appropriate encoding scheme based on a form of Minimum Description Length (MDL) [120]. This is, GRAPHSCOPE seeks for two partitions, one for each part of the bipartite input graph, that minimize the encoding costs with respect to the current graph segment. It computes good partitions in that sense by an iterative greedy approach. Based on the same idea, the MDL is further used to decide whether a newly arriving snapshot belongs to the current graph segment or starts a new segment. If the new snapshot belongs to the current graph segment, the two partitions for the graph segment are updated starting the iteration with the previous partitions. If the new snapshot differs too much from the previous snapshots, a new segment is started. In order to find new partitions in the new segment, the iterative greedy approach is either initialized with the partitions of the previous graph segment or the iterations are done from scratch. The latter can be seen as a static version of GRAPHSCOPE. An experimental comparison on real world data proves a much better running time of the dynamic approach with respect to the static approach. Additional experiments further illustrate that the found source and destination partitions correspond to semantically meaningful clusters. Although this approach 
focuses on bipartite graphs, it can be easily modified to deal with unipartite graphs, by constraining the source partitions to be the same as the destination partitions [30].

Detecting Overlapping Dense Subgraphs in Microblog Streams. The approaches of Angel at al. [8] and Agarwal et al. [1] both date back to the year 2012 and aim at real-time discovery of emerging events in microblog streams, as provided for example by Twitter. To this end, they model the microblog stream as an evolving graph that represents the correlation of keywords occurring in the blogs or messages. In this keyword graph, they seek for groups of highly correlated keywords, which represent events and are updated over the time. Since a keyword may be involved in several events, these groups are allowed to overlap. The main differences between both attempts is the definition of the correlation between keywords and the definition of the desired subgraphs. Angel et al. consider two keywords as correlated if they appear together in the same message. Two keywords are the stronger correlated the more messages contain them together. The messages are considered as a stream and older messages time out. This results in atomic updates of the keyword graph. In contrast, Agarwal et al. consider multiple changes in the keyword graph resulting from a sliding time window. They consider two keywords as correlated if they appear in (possibly distinct) messages of the same user within the time window. Furthermore, they ignore all edges representing a correlation below a given threshold, which results in an unweighted keyword graph.

Regarding the group detection, Angel et al. introduce an algorithm called DYNDENS that considers a parameterized definition of density, which covers most standard density notions. Based on this definition, a set is dense if its density is greater than a given threshold. In order to return all dense subgraphs for each time step, a set of almost dense subgraphs is maintained over time that has the property that after a change in the keyword graph each (possibly new) dense subgraph contains one of the maintained almost dense subgraphs. Hence, the almost dense subgraphs can be iteratively grown to proper density, thus finding all new dense subgraphs after the change. With the help of an appropriate data structure the almost dense subgraphs can be maintained efficiently with respect to time and space requirements. In order to give experimental evidence of the feasibility of their approach, the authors have built a live demo for their techniques on Twitter-tweets and provide, besides a qualitative evaluation, a comparison with a simple static baseline approach that periodically recomputes all dense subgraphs. This static approach took that much time that it was able to compute the set of new events only every 48 to 96 minutes, compared to a real time event identification performed by the dynamic approach. Instead of dense subgraphs, Agarwal et al. seek for subgraphs that possess the property that each edge in the subgraph is part of a cycle of length at most 4 . This property is highly local, and thus, can be updated efficiently. An experimental study on real-world data comparing the dynamic approach to a static algorithm that computes biconnected subgraphs confirms the efficiency of the local updates.

Other Approaches Admitting the Detection of Overlapping Clusters. While two of the following approaches indeed return overlapping clusters, the remaining approaches use a cluster definition that basically admits overlapping clusters, but the algorithmic steps for finding these clusters are designed such that they explicitly avoid overlaps. The most common reason for such an avoidance is the fact that tracking overlapping clusters over 
time is even more difficult than tracking disjoint clusters, which is why most of the existing tracking frameworks require disjoint clusters.

Takaffoli et al. [142] incrementally apply a method inspired by Chen et al. [33] that basically returns overlapping clusters. In order to apply, in a second step, an independent event detection framework [141] that requires disjoint clusters, they however rework this method such that it prevents overlaps. The idea is to greedily grow clusters around core sets that serve as seeds. In doing so the aim is to maximize the ratio of the average internal degree and the average external degree of the vertices in the cluster, only considering vertices with a positive internal and external degree, respectively. The reworking step then allows a vertex to also leave its initial core set, which admits the shrinking of clusters and the prevention of overlapping clusters. For the first snapshot in a dynamic scenario the initial core sets are single vertices (static version), whereas any further snapshot is clustered using the clusters of the previous snapshot as initial core sets (dynamic approach). If a previous cluster decomposes into several connected components in the current snapshot, the authors consider each of the connected components as a seed. Compared to the static version applied to each snapshot independently and also compared to the FACETNET approach [91] (which we introduce in the category of generative models), at least for the Enron network tested by the authors, the dynamic attempt results in a higher average community size and a higher dynamic modularity per snapshot. The latter is a linear combination of the modularity values of the current clustering with respect to the current snapshot and the previous snapshot (see also the quality measures in evolving graphs presented in Section 1.2).

Kim and Han [80] present an evolutionary clustering method, which incorporates temporal smoothness to SCAN [154], a popular adaption of the (static) density-based data clustering approach DBSCAN [50] to graphs. The new idea, compared to the idea of evolutionary clustering by Chakrabarti et al. [31], is that instead of minimizing a cost function that trades off the snapshot quality and the history quality at every time step, the same effect can be achieved by adapting the distance measure in SCAN. As usual for SCAN, the authors define an $\varepsilon$-neighborhood of a vertex with respect to a distance measure, such that the resulting $\varepsilon$-neighborhood consists of core vertices and border vertices. A cluster is then defined as the union of $\varepsilon$-neighborhoods, each of which having size at least $\eta$, that overlap in at least one core vertex. This kind of clusters can be easily found by a BFS in the graph. This initially yields clusters that may overlap in some border vertices. However, by ignoring vertices that are already assigned to a cluster during the BFS, disjoint clusters can be easily enforced. A vertex that is not found to be a member of a cluster, is classified as noise. By iteratively adapting $\varepsilon$ the authors additionally seek for a clustering of high modularity. When a good clustering is found for the current snapshot, temporal smoothness is incorporated by adapting the distance measure that characterizes the $\varepsilon$-neighborhoods in the next time steps allowing for the distance in the current snapshot and the not yet adapted distance in the next snapshot. Finally, the authors also propose a method for mapping the clusters found in consecutive snapshots, based on mutual information. On synthetic networks of variable numbers of clusters the proposed approach outperformed FACETNET [91] with respect to clustering accuracy and running time. 
Another approach that is also based on DBSCAN and is very similar to SCAN, is called DENGRAPH and is presented by Falkowski et al. [54]. In contrast to Kim and Han, Falkowski et al. do not focus on an evolutionary clustering approach, but introduce dynamic update techniques to construct a new clustering after the change of a vertex or an edge in the underlying graph. These updates are done by locally applying again an BFS (as for the static SCAN approach), but just on the vertices that are close to the change, thereby updating the cluster ids. Experiments on the Enron data set suggest that DENGRAPH is quite fast but also relatively strict as it reveals only small, very dense groups while many vertices are categorized as noise. The dynamic DENGRAPH version proposed in [54] returns disjoint clusters, while in [52] Falkowski presents a dynamic version for overlapping clusters.

A dynamic algorithm that is not based on a static clustering algorithm but also produces overlapping clusters is proposed by Cazabet et al. [29]. In each time step, clusters are updated in the following way. First, it is determined if a new seed cluster, i.e., a small clique of constant size has emerged due to edge updates. Then, existing clusters and seed clusters are extended by additional vertices. To that end, for each cluster $C$, two characteristics are maintained. The first of these characteristics corresponds to the average percentage of vertices a vertex in $C$ can reach in its cluster by a path of length 2 . Very similar, the second characteristic corresponds to the average percentage of vertices a vertex in $C$ can reach in its cluster by at least two distinct path of length 2 . A vertex that is not in $C$ may be included into $C$ if, roughly speaking, this improves both of these characteristics. In a last step, clusters that share a certain percentage of vertices with another cluster are discarded. The goal of this approach is not primarily to get good clusterings for each time step but to get a good clustering of the last time step by taking the evolution of the network into account. Nevertheless, the approach per se is capable of clustering dynamic networks in an online scenario. Other overlapping approaches are categorized according to a different focus and are thus described at another point. For an overview on all overlapping approaches presented in this survey see Table 1.

Algorithms Maintaining Auxiliary Structures. The following two approaches consider atomic changes in the given graph and aim at efficiently updating structurally clearly defined clusters, which are obtained by a simple operation on an auxiliary structure. In the first approach, by Duan et al. [48], the auxiliary structure is a graph that represents the overlap of maximal cliques in the input graph, and the final clusters result from the connected components of this graph. In the second approach, by Görke et al. [67], a partial cut tree (or Gomory-Hu tree [64]) is maintained and the clusters result from the subtrees obtained by deleting a designated vertex in this tree. The latter approach further incorporates possibly given edge weights of the input graph.

The dynamic clique-clustering approach of Duan et al. [48] is a dynamic version of the clique percolation method (PCM) of Derényi et al. [41], which is again a special case of a more general clique-clustering framework proposed by Everett and Borgatti [51]. The framework by Everett and Borgatti applies an arbitrary clustering algorithm to a weighted auxiliary graph $H$ that represents the overlap of the maximal cliques in the input graph. In the special case considered by Derényi et al. and Duan et al., the auxiliary graph $H$ just encodes if two maximal cliques (of at least size $k$ ) overlap in at least $k-1$ vertices, and thus, is an unweighted graph. More precisely, $H$ is a graph 
where the maximal cliques in the input graph represent the vertices and two vertices are connected by an edge if and only if the corresponding cliques share at least $k-1$ vertices. As clustering algorithm Derényi et al. and Duan et al. simply choose a DFS, which returns the connected components of $H$, which induce overlapping clusters in the original graph. The running time of this approach is dominated by the computation of maximal cliques, which is exponential in the number of vertices [27]. The proposed dynamic version is then straightforward. For each type of change, the authors give a procedure to update the auxiliary graph $H$ as well as the DFS-tree $T$, which indicates the connected components of $H$. In doing so, the insertion of an edge is the only change where the computation of new maximal cliques becomes necessary in parts of the input graph. All other changes can be handled by updating the overlap of previous cliques and adapting edges in $H$ and $T$. Hence, the more changes in the dynamic input graph are different from edge insertions, the better the dynamic approach outperforms the static approach, which computes all cliques from scratch after each change.

The dynamic cut-clustering approach of Görke et al. [66,67] is a dynamic version of the static cut-clustering algorithm of Flake et al. [56], based on updating a partial Gomory-Hu tree [64] of an extended input graph $G_{\alpha}$. The graph $G_{\alpha}$ is obtained from the input graph $G$ by inserting an artificial vertex $q$ and artificial edges, each having weight $\alpha$, between $q$ and each vertex in $G$. The input parameter $\alpha$ determines the coarseness of the resulting clustering. A Gomory-Hu tree $T$ for $G_{\alpha}$ then is a weighted tree on the vertices of $G_{\alpha}$ that represents a minimum $s$-t-cut for each vertex pair in $G_{\alpha}$. More precisely, deleting an edge $\{s, t\}$ in $T$ decomposes $T$ in two subtrees inducing a minimum $s$-t-cut in the underlying graph. The weight assigned to the deleted edge in $T$ further corresponds to the costs of the induced minimum $s$ - $t$-cut. For two non-adjacent vertices $u$ and $v$ in $T$, the minimum $u$-v-cut is given by a cheapest edge on the path from $u$ to $v$ in $T$. In order to obtain the final complete clustering, the artificial vertex $q$ is deleted from $T$, resulting in a set of subtrees inducing the clusters. Due to the special properties of the minimum $s$ - $q$-cuts that separate the resulting clusters, Flake et al. are able to prove a guarantee (depending on $\alpha$ ) of the intercluster expansion and the intracluster expansion of the resulting clustering, which in general is NP-hard to compute (cp. the cut-based quality measures introduced in Section 1.2). The dynamic version of the cut-clustering algorithm determines which parts of the current Gomory-Hu tree of $G_{\alpha}$ become invalid due to an atomic change in $G$ and describes how to update these parts depending on the type of the atomic change. The result is a cut clustering of the current graph $G$ with respect to the same parameter value $\alpha$ as in the previous time step. The most difficult and also (in theory) most time consuming type of an update is the update after an edge deletion. However, in most real world instances the actual effort for this operation is still low, as shown by an experimental evaluation on real world data. We stress that there also exists another attempt $[123,124]$ that claims to be a dynamic version of the cut clustering algorithm of Flake et al., however, Görke et al. showed that this attempt is erroneous beyond straightforward correction. Doll et al. [47] further propose a dynamic version of the hierarchical cut-clustering algorithm that results from varying the parameter value $\alpha$, as shown by Flake et al. [56].

Spectral Graph Clustering Methods. The main idea of static spectral graph clustering is to find an $r$-dimensional placement of the vertices such that vertices that form a 
cluster in an appropriate clustering with respect to a given objective are close to each other while vertices that are assigned to different clusters are further away from each other. This can be done by considering the spectrum of a variation of the adjacency matrix, like for example the Laplacian matrix in the context of the normalized cut objective [146]. More precisely, many desirable objectives result in optimization problems that are solved by the eigenvectors associated with the top- $r$ eigenvalues of a variation of the adjacency matrix that represents the objective. The rows of the $n$-by- $r$ matrix formed by these eigenvectors then represent $r$-dimensional coordinates of the vertices that favor the objective. The final clustering is then obtained by applying, for example, $k$-means to these data points.

The EvOLSPEC algorithm by Chi et al. [34] conveys this concept to a dynamic scenario by introducing objectives that incorporate temporal smoothness. Inspired by Chakrabarti et al. [31], the authors linearly combine snapshot costs and temporal costs of a clustering at time step $t$, where the temporal costs either describe how well the current clustering clusters historic data in time step $t-1$ or how different the clusterings in time step $t$ and $t-1$ are. For both quality measures, they give the matrices that represent the corresponding objectives, and thus, allow the use of these measures in the context of spectral graph clustering.

Ning et al. [107] show how to efficiently update the eigenvalues and the associated eigenvectors for established objectives if an edge or a vertex in the underlying graph changes. Compared to the static spectral clustering, which takes $O\left(n^{3 / 2}\right)$ time, this linear incremental approach saves a factor of $n^{1 / 2}$. An experimental evaluation of the running times on Web-blog data (collected by the NEC laboratories) confirm this theoretical result. The fact that the updates yield only approximations of the desired values is not an issue, as further experiments on the approximation error and an analysis of the keywords in the found clusters show.

A concept that is closely related to spectral graph clustering is Low-rank approximations of the adjacency matrix of a graph. Tong et al. [143] do not provide a standalone community detection algorithm but a fast algorithm that returns a good low-rank approximation of the adjacency matrix of a graph that requires only few space. Additionally, they propose efficient updates of these matrix approximations that may enable many clustering methods that use low-rank adjacency matrix approximations to also operate on evolving graphs.

Modularity Based Algorithms. All dynamic community detection algorithms based on explicit modularity optimization are modifications of one of three static agglomerative algorithms that greedily optimize modularity.

The first of these static algorithms, commonly called CNM according to its authors Clauset, Newman and Moore [36], is similar to traditional hierarchical clustering algorithms used in data mining, such as single linkage [131]. Starting from a singleton clustering, i.e., a clustering where each cluster consists of exactly one vertex, among all clusterings that can be reached by merging two of the clusters, the one with the best modularity is chosen. This is iterated until modularity cannot be further improved by merging any of the clusters. Although modularity is an inherently global measure, the improvement of the objective function after a merge operation can be easily calculated by only considering the affected clusters. This means that the set of all possible 
merge operations can be maintained in a heap, which leads to a total running time of $O\left(n^{2} \log n\right)$.

Dinh et al. $[44,46]$ evaluate a straightforward dynamization of the CNM algorithm that works as follows. The graph at the first time step is clustered with the static algorithm and the resulting clustering is stored. In the next time step, we first incorporate all changes in the graph. Then, each vertex that is either newly inserted or incident to an edge that has been modified is freed, i.e., it is removed from its cluster and moved to a newly created singleton cluster. To arrive at the final clustering, CNM is used to determine if merging some of the clusters can again improve modularity. The authors call this framework "Modules Identification in Evolving Networks" (MIEN).

Independently, Görke et al. [69] build upon the same idea, but in a more general setting, which results in the algorithm DGLOBAL. There are two variants of this algorithm, the first one based on freeing vertices in the neighborhood of directly affected vertices and the second one based on a backtracking procedure. In the first variant, the subset of freed vertices can be all vertices in the same cluster, vertices within small hop distance or vertices found by a bounded breadth first search starting from the set of affected vertices. In their experiments, considering these slightly larger subsets instead of only directly affected vertices improves modularity and yields a good tradeoff between running time and quality. The second variant not only stores the clustering from the last time step but the whole sequence of merge operations in the form of a dendrogram. A dendrogram is a binary forest where leaves correspond to vertices in the original graph and vertices on higher levels correspond to merge operations. Additionally, if a vertex in the dendrogram is drawn in a level above another vertex, this encodes that the corresponding merge has been performed later in the algorithm. Figure 3a shows an example of a dendrogram produced by the static CNM algorithm whose resulting clustering consists of two clusters. Storing the whole dendrogram across time steps makes backtracking strategies applicable. To update the clustering for the next time step, the backtracking procedure first retracts a minimum number of merges such that certain requirements are met, which depend on the type of change. In case an intracluster edge has been inserted, the requirement is that its incident vertices are in separate clusters after the backtracking procedure. If an intercluster edge is inserted or an intracluster edge deleted, merges are retracted until both affected vertices are in singleton clusters. If an intercluster edge is deleted, the dendrogram stays unchanged. Afterwards, CNM is used to complete this preliminary clustering. Bansal et al. [15] use a similar approach. The main difference is that instead of backtracking merges in the dendrogram, their algorithm repeats all merge operations from the last time step until an affected vertex is encountered. Again, this preliminary clustering is completed with the static CNM algorithm. Figure 3 illustrates the difference between the two approaches. Both studies report a speedup in running time compared to the static algorithm, Görke et al. additionally show that their approach improves smoothness significantly. In the experiments of Bansal et al., quality in terms of modularity is comparable to the static algorithm, while Görke et al. even observe an improvement of quality on synthetic graphs and excerpts of coauthor graphs derived from arXiv. Görke et al. additionally compare the backtracking variant of DGLOBAL to the variant freeing subsets of vertices; for the test instances, backtracking was consistently faster but yielded worse smoothness values. 


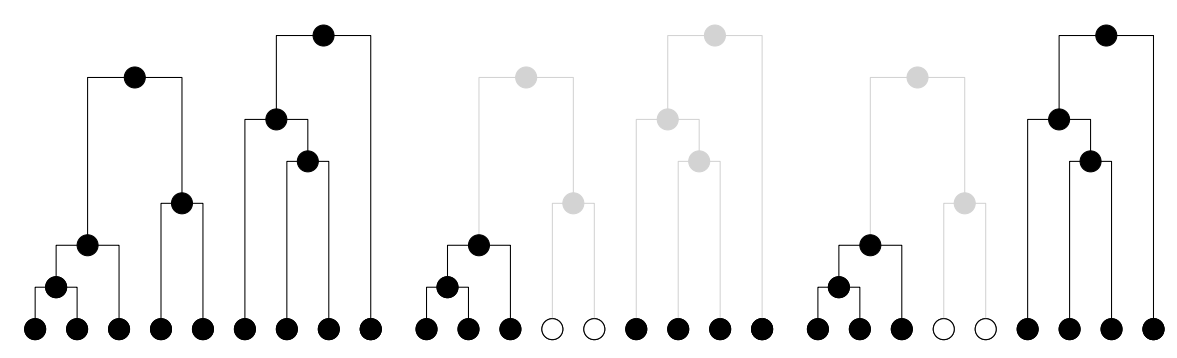

(a) Example dendrogram

(b) Backtracking in [15]

(c) Backtracking in [69]

Fig. 3: Example dendrogram and illustration of backtracking procedure by Görke et al. [69] and Bansal et al. [15] in case an intracluster edge between the white vertices is deleted.

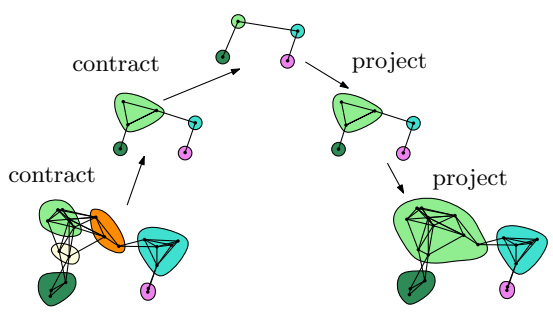

(a) LOUVAIN

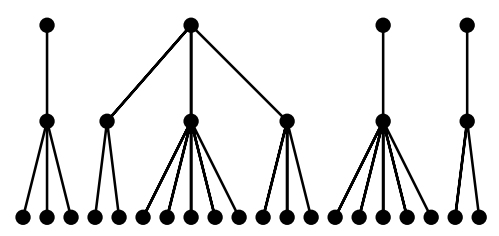

(b) Dendrogram

Fig. 4: Illustration of the LoUVAIN method and the corresponding dendrogram. In the left part, the depicted edge structures show the graphs before the vertex moves, while the colored subsets depict the resulting clusters after the vertex moves on the particular level.

The second static algorithm that has been modified for the dynamic scenario is a local greedy algorithm often called LoUvAIN method [21]. Similar to CNM, the algorithm starts with a singleton clustering. Now, vertices of the graph are considered in a random order. If there is at least one cluster such that moving the current vertex $v$ to it improves the overall modularity, $v$ is moved to the cluster that yields the maximal gain in modularity. This process is repeated in several rounds until a local maximum is attained. Then, clusters are contracted to supernodes and edges between clusters summarized as weighted edges, whereas edges within clusters are mapped to (weighted) self loops. The local moving procedure is then repeated on the abstracted graph taking edge weights into account. Contractions and vertex moves are iterated until the graphs stays unchanged. Then, the clustering is projected down to the lowest level, which represents the original graph, to get the final result. Figure 4 illustrates this procedure.

Among the modifications of the LOUVAIN method to the dynamic scenario, the one by Aynaud and Guillaume [12] is the most direct. In their study, instead of the singleton clustering, the clustering from the last time step is used to initialize the clustering on the 
lowest level. Using a dynamic network of webblogs, they demonstrate that this modification improves smoothness significantly. In terms of modularity, the modified version follows the static version quite well and yields better quality than a reference algorithm based on random walks called WALKTRAP [115]. The authors further propose to use a tradeoff between modularity and smoothness by removing a fixed percentage of randomly chosen vertices from their cluster in each time step, in order to give the algorithm more freedom to perform necessary changes in the clustering.

An evolutionary version of the LOUVAIN method is proposed by Görke et al. [69], called TDLOCAL. Here, the clustering is again reinitialized by the singleton clustering in each time step. Inspired by Chakrabarti et al. [31], smoothness is encouraged by optimizing a linear combination of modularity and the graph theoretic Rand index. It is possible to optimize this modified objective with the LOUVAIN algorithm without increasing the asymptotic running time of one round.

A dynamic version of the LOUVAIN method based on local updates is the algorithm QCA proposed by Nguyen et al. [104]. Depending on the kind of change, the following case distinction is used. If a new vertex $v$ is inserted, $v$ is greedily assigned to a cluster such that modularity is optimized. In case an intercluster edge between $u$ and $v$ is inserted, the algorithm first checks if $u$ or $v$ can be moved to the other cluster such that modularity increases. If yes, it checks if neighbors of the moved vertex can be moved as well. In case a vertex is deleted, its cluster is potentially split by using a method similar to clique percolation [110] restricted to the affected cluster. If an intracluster edge between $u$ and $v$ is deleted in cluster $C$, where $u$ and $v$ have degree at least 2 , the set of maximal quasi-cliques within $C$ is determined and the clustering completed similar to static CNM. In all other cases, the clustering from the last time step is left unchanged.

All of these approaches only maintain one clustering across time steps, namely, the one that could not be improved in terms of modularity. This clustering corresponds to the highest level in the dendrogram. In contrast to that, Görke et al. [69] propose to store and possibly modify the whole dendrogram during the course of the algorithm, which leads to the algorithm framework DLOCAL. After all changes have been incorporated into the graph of the lowest level (the original graph), all affected vertices, i.e., vertices that are either new or incident to edge changes, are marked. Additionally, depending on some policy $P$, some vertices in their close neighbourhood are marked as well. The set of policies evaluated in this study correspond to the aforementioned subset strategies evaluated by Görke et al. [69] for DGLOBAL. This means, $P$ can correspond to freeing vertices within a small hop distance from affected vertices, vertices in the same cluster and vertices found by a bounded breadth first search. Then, vertices on the lowest level are greedily moved until modularity cannot be further improved. Now, affected vertices in the second level of the dendrogram are marked, i.e., subclusters affected by lower level changes. Depending on $P$, again, some vertices in their neighborhood are marked and possibly moved. This is repeated on all levels. The current clustering can be found at all time steps by considering the projection of the highest to the lowest level. Keeping the whole dendrogram in memory instead of only its highest level yields the additional possibility to merge and split clusters on intermediate levels and therefore increases the search space used for local moving, which leads to possibly better quality. Compared to the algorithm DGLOBAL, the experiments of Görke et al. do not give a conclusive 
results; which of these algorithms performs better depends on the structure of the graph and its communities.

An approach that is very similar to DLOCAL is used in the algorithm $\mathrm{A}^{3} \mathrm{CS}$ proposed by Nguyen et al. [43]. The main difference is that the lowest level of the dendrogram is not computed and maintained by a local moving procedure but by an algorithm similar to the static Low-degree Following Algorithm proposed by Dinh and Thai [45]. This algorithm has the nice property to yield approximation guarantees for graphs with a perfect powerlaw degree distribution with a powerlaw exponent larger than 2 . This property is inherited by the dynamic algorithm $\mathrm{A}^{3} \mathrm{CS}$. However, the clusters produced by this algorithm are quite small, which is why it makes sense to additionally consider local moving (which includes merging of clusters) on higher levels to further improve its practical performance. Dinh and Thai compare $\mathrm{A}^{3} \mathrm{CS}$ to QCA and MIEN, with the result that it dominates both with respect to running time and quality.

The third static algorithm for modularity based clustering, which lends itself especially well to parallelization, is based on the contraction of matchings [121, 128]. To effectively optimize modularity, edges are weighted based on the gain in modularity corresponding to the merge of the two adjacent vertices. Using these edge weights, a weighted matching is computed in a greedy fashion, possibly in parallel [119]. Riedy and Bader [118] propose a dynamic variant of this algorithm especially for the case of larger batch sizes, i.e., many changes between consecutive time steps. Across the time steps, the current clustering together with its community graph is stored. After incorporating the edge changes in the community graph, all (elementary) vertices incident to newly inserted intercluster or deleted intracluster edges are extracted from their community. Then, the matching based agglomeration is restarted from the modified community graph. As the community graph is usually much smaller than the original graph, this potentially saves running time.

Label Propagation/Diffusion. An algorithm that is not based on modularity, but conceptually closely related to the LOUVAIN method is LABEL PROPAGATION [116]. LABEL PROPAGATION can be seen as an algorithm greedily maximizing the number of edges within clusters by moving vertices, very similar to the local moving procedure in the LOUVAIN method. Obviously, the global optimum with respect to the number of edges within clusters is trivial, as it corresponds to assigning all vertices to one cluster. Hence, using local moving in a multilevel scheme, as in the case of modularity maximization, does not make much sense. Instead, one is interested in the local maximum obtained after local moving on the original graph, which corresponds to the lowest level in the dendrogram. LABEL PROPAGATION is very fast and lends itself well to parallelization [136]. Xie and Szymanski propose a modified version of this algorithm called LABELRANK [152]. In contrast to the original LABEL PROPAGATION algorithm, each vertex does not maintain one single cluster id or label, but instead a vector of containment probabilities for each cluster currently existing in the graph. Label propagation phases alternate with inflation and cutoff steps to decrease the computational complexity and to make the differences in the particular vectors more pronounced. To prevent the algorithm from converging too fast to the (potentially uninteresting) static distribution, only labels of vertices are updated that are sufficiently different from their neighbors. The algorithm outputs a set of labels for each vertex that each has an associated prob- 
ability, which would lead to overlapping clusters. This is why, although overlaps are resolved in a preprocessing step by only considering the strongest label for each vertex, we list the algorithm both among overlapping and non overlapping approaches in Table 1. Both LABEL PROPAGATION and LABELRANK have been modified to the dynamic scenario $[111,151]$, roughly based on the idea to only update labels/label vectors of vertices affected by changes in the graph. The dynamic version of LABELRANK is called LABELRANKT.

A concept that is very similar to LABELRANK and has been developed in the context of graph partitioning is diffusion [72,94,95]. Similar to the above algorithm, each vertex maintains a vector of size $k$ indicating to which extent it is connected to the vertices of each of the $k$ clusters. The entries of these vectors are called loads; loads are distributed through the network along the edges in rounds, which explains the origin of the name diffusion. Based on this concept, Gehweiler and Meyerhenke [60] propose a distributed graph clustering algorithm called DIDIC, which is motivated by the task to cluster nodes of a peer-to-peer based virtual distributed supercomputer. The weight of edges between nodes in this network corresponds to the bandwidth between the associated peers. The idea is to find clusters of highly connected peers that can be used to solve a common task in parallel. In contrast to LABELRANKT, they use a second diffusion system drawing the loads associated with cluster $i$ back to the vertices in cluster $i$, which accelerates the formation of large, connected clusters. In the first time step, the process starts with a random clustering and distributes the load of each cluster to the vertices it contains. After the diffusion process has been run for a certain number of rounds, clusters are reassigned such that each vertex moves to the cluster from which it obtained the highest load value, leading to a complete clustering. The algorithm is made dynamic by initializing the clusters and load vectors with the values obtained in the previous time step, instead of random initialization.

Generative Models. Another popular approach to clustering problems is the use of generative models that assume the graph is randomly generated on the basis of a hidden ground truth clustering. The goal is now to approximately recover the hidden or latent clustering by looking for clusterings that are likely given the observed outcome of this random process, which corresponds to the given graph. Given conditional probabilities that estimate this likelihood and a prior distribution over the set of all possible clusterings, the posterior probability of a given clustering can be obtained via Bayes' theorem. For conditional probabilities, a common choice are stochastic block models [7, $133,148]$, which generally assume that the probability of a link between two vertices is determined by their cluster membership. If the number of clusters is not provided as an input parameter, a popular choice as a prior distribution for the cluster assignments is the distribution induced by the Chinese restaurant process [6] and its derivations. The goal of maximum a posteriori (MAP) estimations is now to find solutions with high posterior probability.

Among the few approaches based on these concepts that explicitly address the issue of dynamic graphs are FACETNET [91] and the algorithm by Yang et al. [155]. The goal of both approaches is to implicitly enforce smoothness by choosing the prior distribution such that large changes in the clustering between adjacent time steps are assumed to be unlikely. In contrast to traditional stochastic block models, FACETNET builds 
upon the model proposed by Yu et al. [156] that assumes "soft community membership", i.e. vertices belong to different clusters to more or less extent. This results in an overlapping clustering. However, these clusters can easily be converted to a complete clustering in a postprocessing step by assigning each vertex to the cluster it participates in to the largest extent. For this reason and the fact that this is often done when comparing complete clusterings to the clusterings produced by FACETNET, we list the algorithm both under overlapping and non overlapping clusterings in Table 1. In the generative model, the probability of a certain cluster assignment at time step $t$ depends on the cluster assignment at step $t-1$. Depending on a parameter $\nu$, the transitions will be more or less smooth. It can be shown that under certain assumptions, the MAP estimation of this model is equivalent to the framework of Chakrabarti [31]. In this context, the KL-divergence between the observed weight matrix and an approximation of it based on cluster assignments is used as the snapshot cost and the KL-divergence between the clustering at time step $t$ and at time step $t-1$ as history cost. For the inference step, an expectation maximization algorithm is used that is guaranteed to converge towards a locally optimal solution of the corresponding MAP problem. In the FACETNET framework, the number of clusters can change over time. To determine the best number of clusters for each time step, an extension of modularity to soft community memberships is proposed. In the experimental part, synthetic and real world networks are used to evaluate the performance of FACETNET and to compare it to its static counterpart as well as a static and evolutionary (EVOLSPEC) version of spectral clustering [34, 130]. With respect to quality, the FACETNET approach compares favorably.

In the algorithm of Yang et al., the number of clusters is given as input. Given the hidden clustering at a certain time step, the conditional probability for a link between two vertices is determined by the linking probabilities associated with their respective clusters. These linking probabilities are in turn random variables such that their prior distribution causes higher linking probabilities for intracluster edges. The whole generative model corresponds to a Bayesian net where the latent variables associated with a certain time step depend on the clustering from the last time step, a matrix $A$ specifying the probability that a vertex moves from a certain cluster to another in the current time step, and the prior distribution for the linking probabilities between clusters. Again, the prior distribution for $A$ biases the moving probabilities in such a way that the probability for each vertex to move to another community $k$ is smaller than the probability to stay in its own cluster, which implicitly biases the model towards smoothness. The model can be generalized to weighted graphs in a straightforward way. For the inference step, the authors evaluate both the online and the offline scenario. In the online scenario, the variables are sampled from time step to time step using the observations seen so far. In the offline scenario, all variables are sampled together by taking both past and future observations into account. In both cases, a Gibbs sampler is used to infer the latent variables. In the offline scenario, additionally, an expectation maximization algorithm is proposed. These two variants are then compared against each other, against static statistical blockmodels, and the dynamic algorithms EvOLSPEC and FACETNET and their static counterparts. Experiments on synthetic and real world networks suggest that the approach based on Gibbs sampling in combination with the new generative model 
yields the best quality. It might be worth to mention that the hyperparameters that influence the priors are tuned by considering the modularity of the resulting clusterings.

Another generative approach has been used by Sun et al. [138] for their algorithm Evo-NetClus. Similar to FACETNET, Evo-NetClus builds upon soft community membership. In contrast to the models mentioned above, the networks considered are assumed to contain vertices of multiple types, where one of the types is defined as a special "center type". Each edge is incident to exactly one center type vertex; the authors call this star network schema. As an illustrative example that is also used in the experiments, the authors use publication networks as for example DBLP ${ }^{1}$. Here, vertices of the center type correspond to papers and the other types to authors, conferences and associated terms. Again, the probability of the clustering of time step $t$ is influenced by the clustering at time step $t-1$, favoring smoothness. The model incorporates changing cluster numbers in each time steps that are not assumed to be input parameters. For the inference step, an online Gibbs sampler is proposed. With respect to quality, the authors compare their model to degenerated models that do not take historical data or only a subset of types into account.

Further Approaches. In this category we list three approaches that do not fit into any of the previous categories. The first approach considers a bicriterial optimization problem while the former approaches focus on a single criterion, the second approach is a general framework that allows to incorporate temporal smoothness into basically every static clustering algorithm, and the third approach claims that considering the input graph as a homogeneous structure where in each region the same criteria for good clusters hold is not appropriate.

The approach of Kim et al. [79] is based on optimizing two different measures simultaneously in a bicriterial approach. Typically, the measures in bicriterial approaches are competing in the sense that one measure tends towards the 1-clustering and the other towards the singleton clustering. The goal is to approximate the pareto front, i.e., to find a set of clusterings that are not dominated by other clusterings with respect to both criteria. Kim et al. use as criteria (or fitness functions) a global version of MinMaxCut [42], which tends to the 1-clustering, and a global version of the silhouette index [122], which tends to the singleton clustering. They approximate the pareto front by an evolutionary metaheuristic in a dynamic scenario using a locus-based representation of clusterings [112], which is a vector of length $n$ storing for each vertex exactly one outgoing edge. The represented clustering then corresponds to the connected components of the induced graph. The locus-based representation has the advantage that different clusterings can be combined (crossed) in a meaningful way by performing uniform crossover on the corresponding vectors, which means that each entry in the resulting vector is randomly taken from one of the parent vectors. The dynamization is trivially done by initializing the population of the current time step by the result from the last time step. Different evolutionary metaheuristics are compared with respect to both criteria on a dynamic graph representing YouTube videos.

While the former approach uses evolutionary metaheuristics, which have nothing to do with evolutionary clustering according to Chakrabarti et al. [31], the next approach

\footnotetext{
${ }^{1} \mathrm{http} / / / \mathrm{www}$. informatik.uni-trier.de/ ley/db/
} 
is again an evolutionary clustering approach. In contrast to other evolutionary clustering approaches, which most often incorporate temporal smoothness into a particular clustering algorithm, the framework introduced by Xu et al. [153] can be applied with any static clustering method. In their publication the authors use the normalized cut spectral clustering approach by Yu and Shi [157]. Although the idea of Xu et al. is inspired by Chakrabarti et al., the main difference is that they do not incorporate temporal smoothness by optimizing a linear combination of snapshot quality and history quality, but adapt the input data for the chosen clustering algorithm based on the community structure found in the previous snapshot. This adaption is done as follows. The adjacency matrices of the snapshots are considered as realizations of a non stationary random process which allows to define an expected adjacency matrix for the current snapshot. Based on this expected matrix a smoothed adjacency matrix can be approximated that also takes into account the previous time step. The smoothed adjacency matrix is a convex combination of the smoothed adjacency matrix of the previous time step and the actual adjacency matrix of the current time step. The parameter that balances the two terms of the convex combination is estimated such that it minimizes a mean squared error criterion. The chosen clustering algorithm is then applied to the estimated smoothed adjacency matrix, thus incorporating temporal smoothness to stabilize the variation of the found clusters over time.

All the above clustering approaches use the same objective for the whole graph to get good clusterings. In this way these approaches consider the input graph as homogeneous structure, regardless whether parts of the graph are sparser than others, and thus, possibly require another notion of density for reasonable communities than denser parts. Wang et al. [82] follow Aggarwal et al. [2] who claim that considering networks as homogeneous structures is not an appropriate attempt. This is why Wang et al. introduce patterns describing homogeneous regions that are consolidated in a second step to generate non overlapping clusters. In contrast to density, which depends on the number or the weight of edges within a subgraph or cluster, homogeneity means that all vertices in a pattern have similarly weighted neighbors. In order to efficiently compute the patterns in a dynamic scenario, the authors maintain, by incremental updates, a top- $k$ neighbor list and a top- $k$ candidate list as auxiliary structures. These updates are able to deal with atomic changes as well as with several changes (of vertices and edge weights) in one time step. In comparison with FACETNET [91] and the evolutionary clustering method by Kim and Han [80], experiments on the DBLP, the ACM and the IBM data set prove a better processing rate (number of vertices processed per second) and a better accuracy of the found clusterings in locally heterogeneous graphs.

Summary. To provides a summary of all algorithms described in this section, Table 1 lists some of their basic properties. These include whether the authors aim at running time or smoothness, or both, and if the resulting clusterings are overlapping or not. If applicable, we further give a reference to an existing static algorithm the approach is based upon. Among the algorithms we considered, the majority focuses on the task of finding non overlapping clusters. Interesting is that the number of algorithms aiming at low running time is almost the same as the number of algorithms aiming at smoothness; only very few algorithms take both into account. Note that an entry in the table indicating that an algorithm does not aim at low running time or smoothness does not indicate 
that the algorithm is slow or produces unsmooth clusterings; it just signifies that this aspect was neither considered in the conception of the algorithm nor evaluated in the experiments. In general, it is expected that smoothness and running time go together quite well, as the use of local updates often improves both of these aspects.

\section{Data Sets}

In this section, we aim to give an overview on what kind of data sets have been used in current publications regarding the clustering of evolving graphs. In the first part, we concentrate on real world instances, i.e., instances that correspond to data collected from observed relationships between objects or persons. In the second part, we briefly talk about models and generators for evolving networks, with a special focus on synthetic data incorporating a hidden ground truth clustering.

\subsection{Real World instances}

Most networks described in this category are based on human interaction and can therefore be classified as social networks in the wider sense. We tried to assign them to more fine grained subcategories depending on their structure and interpretation.

Email networks. One of the few publicly available networks corresponding to social interaction and containing both time information and additional metadata is the Enron email dataset ${ }^{2}$. It represents the email exchange of employees of the Enron Corporation and was made public during the legal investigation concerning the Enron corporation. According to the information given on the above mentioned website, the dataset contains about 600000 emails belonging to 158 users. Note that the total number of distinct email addresses in the data is much larger, as also emails to and from non-Enron email addresses are recorded. In most network representations of the dataset, employees are modeled as vertices and two vertices are connected by an edge if and only if the dataset contains an email between the two corresponding employees. Since the data also distinguishes between sender and recipient, edges are sometimes directed. Furthermore, the emails of a certain time period are often aggregated in one snapshot. This may result in multiple edges or in weighted edges representing the frequency. Hence, depending on the concrete modeling, different authors refer to quite different dynamic graphs as "the Enron network", which makes comparisons between the experimental findings rather difficult. This is also the case for static data sets; however, due to even more degrees of freedom, for example the frequency of time steps or the question whether relations age and disappear over time, this is even more immanent in the case of dynamic data. Takaffoli et al. [142] choose monthly snapshots over one year, which considerably decreases the number of vertices and edges in the network. As a kind of ground truth clustering, they identify "persisting topics" based on keyword extraction. Duan et al. [48] and Dinh et al. [46, 104] consider emails on a weekly basis and do not consider any metadata. The Enron dataset has also been used in the evaluation of GRAPHSCOPE [137]; however,

\footnotetext{
${ }^{2}$ available at http://www.cs.cmu.edu/ enron/
} 
Table 1: Systematic overview on main features of the clustering algorithms and community detection approaches presented in Section 2.

\begin{tabular}{|c|c|c|c|c|c|}
\hline \multirow[b]{2}{*}{ Reference } & \multicolumn{2}{|c|}{ aims at } & \multicolumn{2}{|c|}{ overlapping } & \multirow{2}{*}{$\begin{array}{l}\text { based on } \\
\text { existing static approach }\end{array}$} \\
\hline & run. time & smoothn. & yes & no & \\
\hline Sun et al. [137] (GRAPHSCOPE) & $\otimes$ & ( ) & $\otimes$ & ( ) & $\otimes$ \\
\hline Angel at al. [8] (DYNDENS) & $(1)$ & $\otimes$ & $(1)$ & $\otimes$ & $凶$ \\
\hline Agarwal et al. [1] & 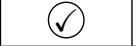 & $\otimes$ & $(\nabla)$ & $\otimes$ & $\otimes$ \\
\hline Takaffoli et al. [142] & $\otimes$ & 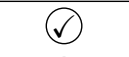 & $(\checkmark)$ & $(\nabla)$ & Chen et al. [33] \\
\hline Kim and Han [80] & $\otimes$ & 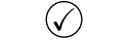 & $(\checkmark)$ & $(\nabla$ & Xu et al. [154] (SCAN) \\
\hline $\begin{array}{l}\text { Falkowski et al. }[54,52] \\
\text { (DENGRAPH) }\end{array}$ & $(\nabla$ & $凶$ & $(\nabla)$ & $\oslash$ & Xu et al. [154] (SCAN) \\
\hline Cazabet et al. [29] & $\otimes$ & $(\nabla)$ & $(\nabla)$ & $\otimes$ & $\otimes$ \\
\hline Duan et al. [48] & $(\theta$ & $\otimes$ & $(\nabla)$ & $\otimes$ & Derényi et al. [41] (PCM) \\
\hline Görke et al. $[66,67]$ & $(0$ & ( & $凶$ & $(\nabla$ & Flake et al. [56] \\
\hline Chi et al. [34] (EvolSPEC) & $\otimes$ & $(1)$ & $\otimes$ & $(\nabla)$ & Shi and Malik [130] \\
\hline Ning et al. [107] & $(0$ & $\otimes$ & $\otimes$ & ( & Shi and Malik [130] \\
\hline Dinh et al. $[44,46]$ (MIEN) & $(1)$ & $\otimes$ & $\otimes$ & $(1)$ & Newman and Moore [36] (CNM) \\
\hline Görke et al. [69] (DGLOBAL) & $(0$ & $(0$ & $\otimes$ & ( ) & Newman and Moore [36] (CNM) \\
\hline Bansal et al. [15] & $(1)$ & $\otimes$ & $\otimes$ & $\oslash$ & Newman and Moore [36] (CNM) \\
\hline Aynaud and Guillaume [12] & $\otimes$ & $\oslash$ & $\otimes$ & $\oslash$ & Blondel et al. [21] (LOUvAIN) \\
\hline Görke et al. [69] (TDLOCAL) & $\otimes$ & $\oslash$ & $\otimes$ & $\oslash$ & Blondel et al. [21] (LouvAin) \\
\hline Nguyen et al. [104] (QCA) & 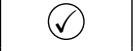 & $\otimes$ & $\otimes$ & $(\nabla)$ & Blondel et al. [21] (Louvain) \\
\hline Görke et al. [69] (DLOCAL) & $(\nabla$ & $\oslash$ & $\otimes$ & 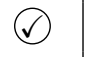 & Blondel et al. [21] (Louvain) \\
\hline Nguyen et al. [43] (A $\left.{ }^{3} \mathrm{CS}\right)$ & $(0$ & $\otimes$ & $\otimes$ & $\oslash$ & $\begin{array}{l}\text { Dinh and Thai [45] and } \\
\text { Blondel et al. [21] (LouvAIN) }\end{array}$ \\
\hline Riedy and Bader [118] & $(1)$ & $\otimes$ & $\otimes$ & $(1)$ & Riedy et al. [119] \\
\hline Pang et al. [111] & $(\theta$ & $\otimes$ & $\otimes$ & $(1)$ & $\begin{array}{l}\text { Raghavan et al. [116] } \\
\text { (LABEL PROPAGATION) }\end{array}$ \\
\hline $\begin{array}{l}\text { Xie et al. [151] } \\
\text { (LABELRANKT) }\end{array}$ & $(1)$ & $\otimes$ & $(\nabla)$ & $(1)$ & Xie et al. [152] (LABELRANK) \\
\hline Gehweiler et al. [60] (DIDIC) & $\oslash$ & $\otimes$ & ( & $(0$ & Meyerhenke et al. [95] \\
\hline Lin et al. [91] (FACETNET) & $\otimes$ & $\oslash$ & $\oslash$ & $(\checkmark)$ & Yu et al. [156] \\
\hline Yang et al. [155] & $\otimes$ & ( ) & $\otimes$ & ( ) & $\otimes$ \\
\hline $\begin{array}{l}\text { Sun et al. [138] } \\
\text { (Evo-NetClus) }\end{array}$ & $\otimes$ & $(\nabla$ & ( & $(\checkmark)$ & Sun et al. [139] (NETClus) \\
\hline Kim et al. [79] & $\otimes$ & $(1)$ & $\otimes$ & $(\nabla$ & $\otimes$ \\
\hline Wang et al. [82] & $(\nabla$ & $凶$ & 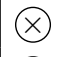 & ( & $凶$ \\
\hline Xu et al. [153] & $\otimes$ & $(0$ & $\otimes$ & ( ) & $\otimes$ \\
\hline
\end{tabular}


there the data is considered as a directed and bipartite graph, where one part corresponds to senders and the other to receivers of emails. Görke et al. [69] did not consider Enron data, but an anonymized network of e-mail contacts at the department of computer science at KIT. It is comprised of about 700000 events collected over a period of about 2.5 years $^{3}$. As metadata, it includes an id for each email address specifying the corresponding chair, which can be considered as ground truth clusters.

Cellphone data. Very similar to email networks are data about phone calls. Palla et al. [109] cluster a network of phone calls between the customers of a mobile phone company containing data of over 4 million users. They consider edges to be weighted; a phone call contributes to the weight between the participating customers for some time period around the actual time of the call. As metadata to evaluate their community finding approach, they consider zip code and age of customers. Similar data is considered by Green et al. [73], however, they do not consider edge weights. The Reality MiningDataset [49] is provided by the MIT Human Dynamics $\mathrm{Lab}^{4}$ and was collected during a social science experiment in 2004. It includes information about call logs, Bluetooth devices in proximity, cell tower IDs, application usage, and phone status of 94 subjects over the course of an academic year. In the context of dynamic graph clustering, it is possible to extract test data in various ways. Xu et al. [153] construct a dynamic graph where the edge weight between two participants in a time step corresponds to the number of intervals in which they were in close physical proximity. As ground truth clustering, they use the affiliations of the participants. Sun et al. [137] additionally consider the cellphone activity to construct a second dynamic graph.

Online social networks and blogs. Another prime example of social networks are online social networks like Facebook or Flickr. In the context of clustering algorithms, they are particularly interesting due to their size and the fact that friendship links are explicit and not implicitly assumed with the help of other metadata. Viswanath et al. [145] crawled the regional network of Facebook in New Orleans. Only data from public profiles is collected, giving information about approximately 63000 users and 1.5 Mio. friendship links, together with their evolution. Nguyen et al. [104] and Dinh et al. [43] use these data to evaluate their clustering algorithms. Kumar et al. [81] analyze data from Flickr and Yahoo! $360^{\circ}$. Whereas Yahoo! $360^{\circ}$ was a typical social network that does not exist anymore, Flickr has a focus on the sharing of photos, although friendship links exist as well. Both datasets are used by the authors in anonymized form and are not publicly available. Closely related to online social networks are networks derived from blogging platforms; here, the edges correspond to entry-to-entry links between different blogs [12,91,107,155]. Angel et al. [8] use sampled data obtained via Twitter's restricted access to its data stream ${ }^{6}$. LiveJournal ${ }^{7}$ is somewhere in between a network of blogs and an online social network. Interesting is that users can explicitly create

\footnotetext{
${ }^{3}$ For further details and for downloading the whole dataset, please visit http://i11www.iti.unikarlsruhe.de/en/projects/spp1307/emaildata

${ }^{4} \mathrm{http} / / /$ realitycommons.media.mit.edu/realitymining.html

${ }^{5} \mathrm{http} / / /$ www.flickr.com/

${ }^{6} \mathrm{https}: / /$ dev.twitter.com/docs/streaming-apis\#sampling

${ }^{7}$ http://www.livejournal.com/
} 
friendship links as well as join groups. In contrast to the usual way dynamic networks are build from blogs, edges do not necessarily correspond to links but can also depend on friendship links. Backstrom et al. [13] study the evolution of communities in LiveJournal using friendship links as edges and group membership as (overlapping) ground truth clustering.

Publication databases. Publication databases can be used to extract dynamic graphs in several ways. Probably the most common approach is to consider coauthor graphs, in which vertices correspond to authors, and two authors are linked if they coauthored at least one publication. Depending on the model, edges are weighted in different ways depending on the number of shared publications and the number of authors on each publication. An orthogonal view on the data yields copaper networks where vertices correspond to papers and links exist if papers have at least one author in common. Both of these network types are simplifications of bipartite author-paper networks that relate authors to their articles. Another possibility is to not take authorship into account but insert (possibly directed) links between articles if one article cites the other, leading to citation networks. It is commonly believed that clusters in all of these networks correspond to different research topics or fields. Due to the fact that publication data is typically not subject to any privacy concerns and their size is reasonably large, they are often used in the evaluation of graph clustering algorithms $[10,12,13,15,43,46$, $48,69,91,104,138,141,151]$. Another advantage is that information about conferences and journals the articles appeared in can be used as metadata to evaluate the resulting clusterings. The temporal aspect in the data stems from the fact that each publication has an associated publication year. The two most often considered databases in the context of clustering are DBLP and arXiv. DBLP collects information about publications in the field of computer science; information about how this data can be downloaded as an $\mathrm{xml}$ file can be found on the corresponding homepage ${ }^{8}$. The arXiv e-print archive ${ }^{9}$ is a repository that stores electronic e-prints, organized in several categories alongside time stamped metadata. To evaluate their dynamic graph clustering framework, Görke et al. [69] used a dataset obtained from this repository, which can be found, together with the source code of the crawler used to extract this data, on the corresponding project page ${ }^{10}$. The KDD cup 2003 also provides further arXiv datasets on its project page $^{11}$; these have been used to evaluate algorithms in the context of modularity based clustering $[43,46,104]$.

Small examples. Many publications about static graph clustering include the analysis of small networks to illustrate some properties of the clusterings produced by their algorithm. A famous example for that is the karate network collected by Zachary in 1977 [158], which describes friendship links between members of a karate club before the club split up due to an internal dispute; a typical question is whether a clustering algorithm is able to predict the split given the network structure. Usually these networks are small enough to be visualized entirely in an article, which enables readers to

\footnotetext{
${ }^{8}$ http://www.informatik.uni-trier.de/ ley/db/

${ }^{9}$ http://arxiv.org/

${ }^{10}$ http://i11www.iti.uni-karlsruhe.de/en/projects/spp1307/dyneval

${ }^{11} \mathrm{http} / / /$ www.cs.cornell.edu/projects/kddcup/datasets.html
} 
compare different clusterings of these networks across several publications. The closest evolving analog to Zachary's karate network is the Southern Women data set collected in 1933 by Davis et al. [39]. It contains data on the social activities of 18 women observed over a nine-month period. Within this period, they recorded for 14 informal events whether these women participated or not. It has been used as a test set by Berger et al. [17], Berger-Wolf and Saia [18], and Yang et al. [155]. Another interesting small example is Grevi's zebra data set [140] used by Berger et al. [17]. It consists of information about the spatial proximity of members of a zebra herd observed over three months, corresponding to 44 observations or time steps.

Other Data Sets. In the following, we will list some further sources for dynamic graph data already used to evaluate dynamic graph clustering algorithms. Xie et al. [151] have used graphs representing the topology of the internet at the level of autonomous systems (AS Graphs) based on data collected by the University of Oregon Route Views Project [90]. These data are available from the Stanford Large Network Dataset Collection $^{12}$. Xu et al. [153] try to identify communities of spammers in data from Project Honey $\operatorname{Pot}^{13}$, an ongoing project to identify spammers. Sun et al. [138] use data extracted from the social bookmarking web service Delicious ${ }^{14}$, which naturally comes with a plenitude of metadata. Kim et al. [79] use data from youtube crawls ${ }^{15}$ in their evaluation. Pang et al. [111] cluster a dynamic network of players of World of Warcraft, where edges are based on the information whether they take part in the same group.

Static Networks with artificial dynamics. Apart from real world data with a naturally given temporal evolution, it is also possible to artificially incorporate some dynamics into originally static data. Riedy et al. [118], for example, consider static real world networks that become dynamic by generating random edge deletions and insertions.

\subsection{Dynamic Graph Generators}

Depending on the aim of designing a certain clustering algorithm, there are good reasons to use synthetic data as well as good reasons to use not only synthetic data for the evaluation. Synthetic data means graphs that are artificially generated by the help of a graph generator. Given a number of vertices, these generators decide which vertices are connected by an edge based on the probability of such an edge. The edge probabilities are derived for example from a preferential attachment process, where vertices that already have a high degree are connected with higher probability than others, or from other rules that are characteristic for the particular generator. In the context of evolving graphs, graph generators usually not only have to decide which vertices are linked but also which vertices or edges are added or deleted. Furthermore, if the generator incorporates a hidden ground truth clustering, this usually evolves randomly as well, which in turn influences edge probabilities.

\footnotetext{
${ }^{12} \mathrm{http}: / /$ snap.stanford.edu/data/index.html

${ }^{13} \mathrm{http} / / /$ www.projecthoneypot.org/

${ }^{14}$ https://delicious.com/

${ }^{15}$ http://netsg.cs.sfu.ca/youtubedata/
} 
One reason to include real world instances, i.e., instances that stem from typical applications, in the experimental evaluation is that they frequently exhibit very specific properties and symmetries that are difficult to analyze and rebuild in synthetic data. Hence, to predict the performance of an algorithm in a certain application, using only synthetic data is unrewarding, since experiments involving sample instances stemming from this application are often more accurate.

This raises the question of why to use synthetic data at all. There are some good arguments that justify the use of synthetic data, at least together with real world data:

- Tunable characteristics, as for example the density of the generated graphs, allow to evaluate algorithms in detail depending on these characteristics. A scenario where this can be useful is when an algorithm yields good results for some networks but bad results on others. A study on a large set of generated graphs might help to identify characteristics of the graph that are difficult to handle for the algorithm, which in turn might raise some potential for improvements.

- Synthetic graphs can usually be generated in any possible size, even very large networks that might not (yet) exist in practice. This is especially useful in the context of scalability studies.

- Using a graph generator, an unlimited number of different networks with similar properties can be generated, preventing algorithms to focus only on very few benchmark instances. This permits to test algorithms on a representative sample of the graph class one is interested in, ensuring some degree of significance.

- In particular in the context of graph clustering, there is another reason why synthetic networks are quite popular. Since there is no general agreement on a single objective function evaluating the goodness of the clustering, a common approach to evaluate graph clusterings independent of any objective function is the comparison to a known ground truth clustering. The downside of this is that real world graphs with a well-motivated ground truth clustering are still rare. For this reason, synthetic networks incorporating a hidden ground truth clustering that has been used in the generation process are popular.

In the following, we aim to give a short overview of models for synthetic graphs that might be useful in the context of clustering evolving networks. We start with some models especially suited for complex networks that can for example be derived by observing human interaction, with a particular focus on models that try to explain their evolution. In the second part, we give an overview on synthetic benchmark instances that incorporate a hidden ground-truth clustering, together with existing approaches to make these benchmarks dynamic.

Probably the most fundamental model for synthetic graphs are graphs where every edge exists with a fixed, constant probability, as first considered by Gilbert [61] in 1959. Until then, a lot of effort has been put into alternative models that better capture the properties of real world complex networks which typically exhibit characteristics like small diameter, high clustering coefficient and a powerlaw degree distribution [100]. Two classical models are small world networks [150] that explicitly address the first two issues and the Barabási-Albert model [16] that mostly addresses the degree distribution. The latter can be seen as a dynamic model for graph growth according to a preferential attachment process. Numerous variations thereof exist, most of which are targeted 
in capturing more accurately properties observed in real world social networks [81, 90, 144]. Leskovec et al. [89] determine automatically, among a set of parameterized models, the one fitting a set of four large online networks best based on the associated likelihood values. Similarly, Patro et al. [113] propose to use an evolutionary algorithm to choose among a set of parameterized models of network growth the one fitting a given set of input characteristics best, in order to automatically learn the best model for different graph classes.

Although these models incorporate network growth and already reflect common properties of observed complex networks as for example online social networks very well, they do not come with a well motivated inherent ground truth clustering that can be used to evaluate clustering algorithms. An exception to this is the model by Zheleva et at. [160] that is especially targeted at modeling the growth of social networks where vertices can additionally choose to enter special groups of interest. Here, the assumption is that both the network and the group structure evolve simultaneously, influencing each other. It might be possible to use the group structure chosen by the vertices as a ground truth clustering for overlapping clusters, although the group structure is correlated to the network only to a certain extent. In the model of Bagrow [14], starting from a graph generated according to preferential attachment, edges are randomly rewired to incorporate a given ground truth clustering. While this approach combines a ground truth clustering with a realistic degree distribution, the evolution stemming from the preferential attachment process is lost.

For static graph clustering, two synthetic benchmark sets have been used very frequently in the literature; the GN benchmark introduced by Girvan and Newman [62] and the LFR benchmark introduced by Lancichinetti et al. [85]. The GN benchmark is based on the planted partition model [25,37,59], also called ad hoc model, that takes as input a given ground truth clustering and two parameters $p_{\text {in }}$ and $p_{\text {out }}$ that correspond to the linking probabilities between vertices within the same or different clusters. Typically, the GN benchmark is used to determine how well an algorithm is able to recover the ground truth clustering, depending on the gap between $p_{\text {in }}$ and $p_{\text {out }}$. The planted partition model has been generalized to weighted [55] and bipartite [74] graphs as well as hierarchical [161] and overlapping [126] ground truth clusterings. Closely related are relaxed caveman graphs $[4,149]$. Among the dynamic graph clustering algorithms described here, FACETNET [91], the approaches by Yang et al. [155] and Kim and Han [80], and the algorithm framework by Görke et al. [69] have been evaluated with the aid of planted partition graphs. The former two evaluations use graphs from the GN benchmark and introduce dynamics based on vertex moves; in each time step, a constant fraction of vertices leave their cluster and move to a random one. Kim and Han additionally consider a dynamic network that also incorporates the forming and dissolving of clusters and vertex addition and deletion. In contrast to that, Görke et al. use a custom graph generator based on the planted partition model that introduces dynamics by splitting and merging clusters in the ground truth clustering [71]. In each time step, one edge or vertex is added or deleted according to the probabilities prescribed by the current ground truth clustering. Hence, the actual graph structure follows the ground truth clustering with some delay. They also provide an efficient implementation of this generator [68]. 
In the LFR benchmark, cluster sizes as well as vertex degrees are expected to follow a power law distribution. Similar to the planted partition model, vertices share a certain fraction of their links with other vertices in their cluster and the remaining links with random vertices in other parts of the graph. The LFR benchmark has been generalized to weighted and directed graphs, as well as to overlapping clusters [83]. Among the clustering algorithms described in Section 2, Dinh et al. [43] have used a modification of this benchmark to a dynamic setting, whereas Cazabet et al. [29] only use it in a static scenario. Green et al. [73] use dynamic benchmarks based on LFR graphs that incorporate different cluster events, including membership switching, cluster growth, shrinkage, birth and death, and the merge and split of clusters. After the ground truth clustering has been adapted, a new random graph is drawn according to the mechanisms of the LFR benchmark, which results in large differences between adjacent timesteps.

Aldecoa and Marín [5] finally suggest to interpolate between two graphs with a significant clustering structure by rewiring edges at random. This is proposed as an alternative to benchmarks like the GN or LFR benchmark in the context of static clustering algorithms. Here, the assumption is that clusterings of the intermediate states of the graph during the rewiring process should have low distance to both the ground truth clustering of the initial and the final state. The rewiring process could be seen as a model for community evolution. In the context of tracking clusterings over time, Berger et al. [17] do not consider models for dynamic graphs but two scenarios for the evolution of clusters that are more sophisticated than random vertex moves or cluster splits and merges. It remains to mention that, in principle, all generative models used to infer clusterings via a Bayesian approach discussed in Section 2 might also be used as benchmark instances, as they naturally come with a dynamic ground truth clustering.

\subsection{Summary}

Nowadays, a lot of large real world networks have been collected and made available by projects like the Stanford Large Network Dataset Collection ${ }^{16}$. One problem in the context of evaluating clustering algorithms for evolving networks is that even if the original data itself has a temporal aspect, this information is often missing in the thereof constructed networks readily provided in many benchmark sets. On the other hand, the listing in Section 3.1 reveals that there is no real lack of dynamic data that is publicly available. A downside of these data is that converting them to dynamic graphs is often laborious and leaves many degrees of freedom. As discussed in the context of the Enron network, data from the same origin can lead to quite different dynamic graphs, depending on the design choices taken. This makes the comparison of results across different publications cumbersome. For static graph clustering, a set of very frequently used networks mostly taken from the websites of Newman ${ }^{17}$ and Arenas ${ }^{18}$ gained some popularity in the orbit of modularity based methods. It would be nice to have a similar set of common benchmark graphs that are evolving over time. A related issue arises in the context of synthetic benchmarks that incorporate a ground truth clustering. Although a lot of publications about the static case exist, there is still no general agreement

\footnotetext{
${ }^{16} \mathrm{http}: / /$ snap.stanford.edu/data/

${ }^{17} \mathrm{http}: / /$ www-personal.umich.edu/ mejn/netdata/

${ }^{18} \mathrm{http}: / /$ deim.urv.cat/ aarenas/data/welcome.htm
} 
on how to make these data dynamic and what realistic dynamic changes in the ground truth clustering might look like.

\section{Conclusion}

Clustering evolving networks is at least as difficult as clustering static networks since it inherits all the difficulties from the static case and is further faced with additional problems that arise from the evolution of the considered networks. The difficulties inherited from static graph clustering are the many different ideas of what a good clustering is and what a good clustering algorithm is supposed to do, as well as the absence of approved benchmark instances to evaluate and compare the performance of clustering algorithms. Additional tasks arise whenever we seek for temporal smoothness or want to detect and visualize the evolution of clusters over time. Among the vast number of algorithms designed for detecting clusters in evolving graphs, in this survey we only considered graph clustering approaches in online scenarios with an algorithmic focus on the exploitation of structural information from previous time steps. We presented several state-of-the-art algorithms in different categories and summarized the main features of these algorithms in Table 1. As a first step towards common benchmark sets for the evaluation of clustering algorithms also in evolving networks, we explicitly listed data and graph generators that were used by the authors of the publications presented in this survey. With this list we aim at viewing the variety of available data and providing a collection to other authors in order to help them finding reasonable test instances for their particular algorithm. Furthermore, we discussed tasks like cluster mapping, event detection and visualization, which make the found cluster information beneficial for further analysis. We gave a brief overview on state-of-the-art approaches solving also these problems and gave some further references where the reader can find more information regarding these issues.

\section{References}

1. Manoj K. Agarwal, Krithi Ramamritham, and Manish Bhide. Real time discovery of dense clusters in highly dynamic graphs: identifying real world events in highly dynamic environments. In Proceedings of the 38th International Conference on Very Large Databases (VLDB 2012), pages 980-991, 2012.

2. Charu C. Aggarwal, Yan Xie, and Philip S. Yu. Towards community detection in locally heterogeneous networks. In Proceedings of the fifth SIAM International Conference on Data Mining, pages 391-402. SIAM, 2011.

3. Charu C. Aggarwal, Yuchen Zhao, and Philip Yu. A framework for clustering massive graph streams. Statistical Analysis and Data Mining, 3(6):399-416, 2010.

4. Rodrigo Aldecoa and Ignacio Marín. Deciphering Network Community Structure by Surprise. PLoS ONE, 6:e24195, September 2011.

5. Rodrigo Aldecoa and Ignacio Marín. Closed benchmarks for network community structure characterization. Physical Review E, 85:026109, February 2012.

6. David J. Aldous. Exchangeability and related topics. In P. L. Hennequin, editor, École d'Éte de Probabilités de Saint-Flour XIII-1983, volume 1117 of Lecture Notes in Mathematics, pages 1-198. Springer, 1985. 
7. Carolyn J. Anderson, Stanley Wasserman, and Katherine Faust. Building stochastic blockmodels. Social Networks, 14:137-161, 1992.

8. Albert Angel, Nikos Sarkas, Nick Koudas, and Divesh Srivastava. Dense subgraph maintenance under streaming edge weight updates for real-time story identification. Proceedings of the VLDB Endowment, 5(6):574-585, February 2012.

9. Alex Arenas, Jordi Duch, Alberto Fernandez, and Sergio Gomez. Size reduction of complex networks preserving modularity. New Journal of Physics, 9(176), 2007.

10. Sitaram Asur, Srinivasan Parthasarathy, and Duygu Ucar. An event-based framework for characterizing the evolutionary behavior of interaction graphs. ACM Transactions on Knowledge Discovery from Data, 3(4):16:1-16:36, December 2009.

11. Thomas Aynaud, Eric Fleury, Jean-Loup Guillaume, and Qinna Wang. Communities in Evolving Networks: Definitions, Detection, and Analysis Techniques. In Animesh Mukherjee, Monojit Choudhury, Fernando Peruani, Niloy Ganguly, and Bivas Mitra, editors, Dynamics On and Of Complex Networks, volume 2 of Modeling and Simulation in Science, Engineering and Technology, pages 159-200. Springer, 2013.

12. Thomas Aynaud and Jean-Loup Guillaume. Static community detection algorithms for evolving networks. In Proceedings of the 8th Intl. Symposium on Modeling and Optimization in Mobile, Ad Hoc, and Wireless Networks (WiOpt'10), pages 513-519. IEEE Computer Society, 2010.

13. Lars Backstrom, Dan Huttenlocher, Jon M. Kleinberg, and Xiangyang Lan. Group formation in large social networks: membership, growth, and evolution. In Proceedings of the 12th ACM SIGKDD International Conference on Knowledge Discovery and Data Mining, pages 44-54. ACM Press, 2006.

14. James Bagrow. Evaluating local community methods in networks. Journal of Statistical Mechanics: Theory and Experiment, page P05001, 2008. doi:10.1088/17425468/2008/05/P05001.

15. Shweta Bansal, Sanjukta Bhowmick, and Prashant Paymal. Fast Community Detection for Dynamic Complex Networks. In Luciano F. Costa, Alexandre Evsukoff, G. Mangioni, and Ronaldo Menezes, editors, Complex Networks, volume 116 of Communications in Computer and Information Science, pages 196-207. Springer, 2011.

16. Albert-László Barabási and Réka Albert. Emergence of scaling in random networks. Science, 286:509-512, 1999.

17. Tanya Berger-Wolf, David Kempe, and C. Tantipathananandth. A Framework For Community Identification in Dynamic Social Networks. In Proceedings of the 13th ACM SIGKDD International Conference on Knowledge Discovery and Data Mining. ACM Press, 2007.

18. Tanya Berger-Wolf and Jared Saia. A Framework for Analysis of Dynamic Social Networks. In Proceedings of the 12th ACM SIGKDD International Conference on Knowledge Discovery and Data Mining, pages 523-528. ACM Press, 2006.

19. Charles-Edmond Bichot and Patrick Siarry, editors. Graph Partitioning. Wiley, 2011.

20. Cemal Cagatay Bilgin and Bülent Yener. Dynamic Network Evolution: Models, Clustering, Anomaly Detection. Technical report, Rensselaer University, NY, 2008.

21. Vincent Blondel, Jean-Loup Guillaume, Renaud Lambiotte, and Etienne Lefebvre. Fast unfolding of communities in large networks. Journal of Statistical Mechanics: Theory and Experiment, 2008(10), 2008.

22. Petko Bogdanov, Misael Mongiovi, and Ambuj K. Singh. Mining Heavy Subgraphs in Time-Evolving Networks. In Proceedings of the 2011 IEEE International Conference on Data Mining, pages 81-90. IEEE Computer Society, 2011.

23. Karsten M. Borgwardt, Hans-Peter Kriegel, and Peter Wackersreuther. Pattern Mining in Frequent Dynamic Subgraphs. In Proceedings of the 2006 IEEE International Conference on Data Mining, pages 818-822. IEEE Computer Society, 2006. 
24. Ulrik Brandes, Daniel Delling, Marco Gaertler, Robert Görke, Martin Höfer, Zoran Nikoloski, and Dorothea Wagner. On Modularity Clustering. IEEE Transactions on Knowledge and Data Engineering, 20(2):172-188, February 2008.

25. Ulrik Brandes, Marco Gaertler, and Dorothea Wagner. Experiments on Graph Clustering Algorithms. In Proceedings of the 11th Annual European Symposium on Algorithms (ESA'03), volume 2832 of Lecture Notes in Computer Science, pages 568-579. Springer, 2003.

26. Ulrik Brandes, Marco Gaertler, and Dorothea Wagner. Engineering Graph Clustering: Models and Experimental Evaluation. ACM Journal of Experimental Algorithmics, 12(1.1):126, 2007.

27. Coen Bron and Joep A. G. M. Kerbosch. Algorithm 457: Finding all cliques of an undirected graph. Communications of the ACM, 16(9):575-577, 1973.

28. Umit Catalyurek, Erik Boman, Karen Devine, Doruk Bozdag, Robert Heaphy, and Lee Ann Riesen. Hypergraph-based Dynamic Load Balancing for Adaptive Scientific Computations. In 21th International Parallel and Distributed Processing Symposium (IPDPS'07), pages 1-11. IEEE Computer Society, 2007.

29. Rémy Cazabet, Frédéric Amblard, and Chihab Hanachi. Detection of overlapping communities in dynamical social networks. In Proceedings of the 2010 IEEE Second International Conference on Social Computing, pages 309-314. IEEE, 2010.

30. Deepayan Chakrabarti. AutoPart: Parameter-Free Graph Partitioning and Outlier Detection. In Proceedings of the 8th European Conference on Principles and Practice of Knowledge Discovery in Databases, pages 112-124. ACM Press, 2004.

31. Deepayan Chakrabarti, Ravi Kumar, and Andrew S. Tomkins. Evolutionary Clustering. In Proceedings of the 12th ACM SIGKDD International Conference on Knowledge Discovery and Data Mining, pages 554-560. ACM Press, 2006.

32. Jiyang Chen, Justin Fagnan, Randy Goebel, Reihaneh Rabbany, Farzad Sangi, Mansoureh Takaffoli, Eric Verbeek, and Osmar R. Zaïane. Meerkat: Community Mining with Dynamic Social Networks. In Proceedings in the 10th IEEE International Conference on Data Mining - Workshops, pages 1377-1380. IEEE Computer Society, December 2010.

33. Jiyang Chen, Osmar R. Zaïane, and Randy Goebel. Detecting Communities in Large Networks by Iterative Local Expansion. In Proceedings of the 2009 IEEE International Conference on Computational Aspects of Social Networks, pages 105-112. IEEE Computer Society, 2009.

34. Yun Chi, Xiaodan Song, Dengyong Zhou, Koji Hino, and Belle L. Tseng. Evolutionary spectral clustering by incorporating temporal smoothness. In Proceedings of the 13th ACM SIGKDD International Conference on Knowledge Discovery and Data Mining, pages 153162. ACM Press, 2007.

35. Aaron Clauset. Finding local community structure in networks. Physical Review E, 72(2):026132, August 2005.

36. Aaron Clauset, Mark E. J. Newman, and Cristopher Moore. Finding community structure in very large networks. Physical Review E, 70(066111), 2004.

37. Anne Condon and Richard M. Karp. Algorithms for Graph Partitioning on the Planted Partition Model. Randoms Structures and Algorithms, 18(2):116-140, 2001.

38. George Cybenko. Dynamic load balancing for distributed memory multiprocessors. Journal of Parallel and Distributed Computing, 7(2):279-301, October 1989.

39. A. Davis, B.B. Gardner, and M. R. Gardner. Deep South. University of Chicago Press, 1941.

40. Daniel Delling, Marco Gaertler, Robert Görke, and Dorothea Wagner. Engineering Comparators for Graph Clusterings. In Proceedings of the 4th International Conference on Algorithmic Aspects in Information and Management (AAIM'08), volume 5034 of Lecture Notes in Computer Science, pages 131-142. Springer, June 2008. 
41. Imre Derényi, Gergely Palla, and Tamás Vicsek. Clique Percolation in Random Networks. Physical Review Letters, 94:160202, 2005.

42. Chris H. Q. Ding, Xiaofeng He, Hongyuan Zha, Ming Gu, and Horst D. Simon. A Minmax Cut Algorithm for Graph Partitioning and Data Clustering. In Proceedings of the 2001 IEEE International Conference on Data Mining, pages 107-114. IEEE Computer Society, 2001.

43. Thang N. Dinh, N. P. Nguyen, and My T. Thai. An Adaptive Approximation Algorithm for Community Detection in Dynamic Scale-free Networks. In Proceedings of the 32th Annual Joint Conference of the IEEE Computer and Communications Societies (Infocom). IEEE Computer Society Press, 2013. to appear.

44. Thang N. Dinh, Incheol Shin, Nhi K. Thai, My T. Thai, and Taieb Znati. A General Approach for Modules Identification in Evolving Networks. In Michael J. Hirsch, Panos M. Pardalos, and Robert Murphey, editors, Dynamics of Information Systems, volume 40 of Springer Optimization and Its Applications, pages 83-100. Springer, 2010.

45. Thang N. Dinh and My T. Thai. Community Detection in Scale-Free Networks: Approximation Algorithms for Maximizing Modularity. IEEE Journal on Selected Areas in Communications, 31(6):997-1006, 2013.

46. Thang N. Dinh, Xuan Ying, and My T. Thai. Towards social-aware routing in dynamic communication networks. In In Proceedings of the 28th International Performance Computing and Communications Conference (IPCCC), pages 161-168, 2009.

47. Christof Doll, Tanja Hartmann, and Dorothea Wagner. Fully-Dynamic Hierarchical Graph Clustering Using Cut Trees. In Frank Dehne, John Iacono, and Jörg-Rüdiger Sack, editors, Algorithms and Data Structures, 12th International Symposium (WADS'11), volume 6844 of Lecture Notes in Computer Science, pages 338-349. Springer, August 2011.

48. Dongsheng Duan, Yuhua Li, Ruixuan Li, and Zhengding Lu. Incremental K-clique clustering in dynamic social networks. Artificial Intelligence, 38(2):129-147, August 2012.

49. Nathan Eagle and Alex Pentland. Reality mining: sensing complex social systems. Journal Personal and Ubiquitous Computing, 10(4):255-268, March 2006.

50. Martin Ester, Hans-Peter Kriegel, Jörg Sander, and Xiaowei Xu. A Density-Based Algorithm for Discovering Clusters in Large Spatial Databases with Noise. In Proceedings of the 2nd ACM SIGKDD international conference on Knowledge discovery and Data Mining, pages 226-231. ACM Press, 1996.

51. Martin G. Everett and Stephen P. Borgatti. Analyzing clique overlap. Connections, 21(1):49-61, 1998.

52. Tanja Falkowski. Community Analysis in Dynamic Social Networks. PhD thesis, Otto-vonGuericke-Universität Magdeburg, 2009.

53. Tanja Falkowski, Jörg Bartelheimer, and Myra Spiliopoulou. Mining and Visualizing the Evolution of Subgroups in Social Networks. In IEEE/WIC/ACM International Conference on Web Intelligence, pages 52-58. IEEE, 2006.

54. Tanja Falkowski, Anja Barth, and Myra Spiliopoulou. DENGRAPH: A Density-based Community Detection Algorithm. In IEEE/WIC/ACM International Conference on Web Intelligence, pages 112-115. IEEE, 2007.

55. Ying Fan, Menghui Li, Peng Zhang, Jinshan Wu, and Zengru Di. Accuracy and precision of methods for community identification in weighted networks. Physica A, 377(1):363-372, 2007.

56. Gary William Flake, Robert E. Tarjan, and Kostas Tsioutsiouliklis. Graph Clustering and Minimum Cut Trees. Internet Mathematics, 1(4):385-408, 2004.

57. Santo Fortunato. Community detection in graphs. Physics Reports, 486(3-5):75-174, 2010.

58. Santo Fortunato and Marc Barthélemy. Resolution limit in community detection. Proceedings of the National Academy of Science of the United States of America, 104(1):36-41, 2007. 
59. Marco Gaertler, Robert Görke, and Dorothea Wagner. Significance-Driven Graph Clustering. In Proceedings of the 3rd International Conference on Algorithmic Aspects in Information and Management (AAIM'07), Lecture Notes in Computer Science, pages 11-26. Springer, June 2007.

60. Joachim Gehweiler and Henning Meyerhenke. A Distributed Diffusive Heuristic for Clustering a Virtual P2P Supercomputer. In Proc. 7th High-Performance Grid Computing Workshop (HGCW'10) in conjunction with 24th Intl. Parallel and Distributed Processing Symposium (IPDPS'10), pages 1-8. IEEE Computer Society, 2010.

61. Horst Gilbert. Random Graphs. The Annals of Mathematical Statistics, 30(4):1141-1144, 1959.

62. Michelle Girvan and Mark E. J. Newman. Community structure in social and biological networks. Proceedings of the National Academy of Science of the United States of America, 99(12):7821-7826, 2002.

63. Peter A. Gloor and Yan Zhao. TeCFlow - A Temporal Communication Flow Visualizer for Social Network Analysis. In ACM CSCW Workshop on Social Networks, 2004.

64. Ralph E. Gomory and T.C. Hu. Multi-terminal network flows. Journal of the Society for Industrial and Applied Mathematics, 9(4):551-570, December 1961.

65. Robert Görke. An Algorithmic Walk from Static to Dynamic Graph Clustering. PhD thesis, Fakultät für Informatik, February 2010.

66. Robert Görke, Tanja Hartmann, and Dorothea Wagner. Dynamic Graph Clustering Using Minimum-Cut Trees. In Frank Dehne, Marina Gavrilova, Jörg-Rüdiger Sack, and Csaba D. Tóth, editors, Algorithms and Data Structures, 11th International Symposium (WADS'09), volume 5664 of Lecture Notes in Computer Science, pages 339-350. Springer, August 2009.

67. Robert Görke, Tanja Hartmann, and Dorothea Wagner. Dynamic Graph Clustering Using Minimum-Cut Trees. Journal of Graph Algorithms and Applications, 16(2):411-446, 2012.

68. Robert Görke, Roland Kluge, Andrea Schumm, Christian Staudt, and Dorothea Wagner. An Efficient Generator for Clustered Dynamic Random Networks. In Proceedings of the 1st Mediterranean Conference on Algorithms, pages 219-233. Springer, 2012.

69. Robert Görke, Pascal Maillard, Andrea Schumm, Christian Staudt, and Dorothea Wagner. Dynamic Graph Clustering Combining Modularity and Smoothness. ACM Journal of Experimental Algorithmics, 18(1):1.5:1.1-1.5:1.29, April 2013.

70. Robert Görke, Andrea Schumm, and Dorothea Wagner. Density-Constrained Graph Clustering. In Frank Dehne, John Iacono, and Jörg-Rüdiger Sack, editors, Algorithms and Data Structures, 12th International Symposium (WADS'11), volume 6844 of Lecture Notes in Computer Science, pages 679-690. Springer, August 2011.

71. Robert Görke and Christian Staudt. A Generator for Dynamic Clustered Random Graphs. Technical report, iti_wagner, 2009. Informatik, Uni Karlsruhe, TR 2009-7.

72. Leo Grady and Eric 1. Schwartz. Isoperimetric Graph Partitioning for Image Segmentation. IEEE Transactions on Pattern Analysis and Machine Intelligence, 28(3):469-475, 2006.

73. Derek Greene, Dónal Doyle, and Pádraig Cunningham. Tracking the Evolution of Communities in Dynamic Social Networks. In Proceedings of the 2010 IEEE/ACM International Conference on Advances in Social Networks Analysis and Mining, pages 176-183. IEEE Computer Society, 2010.

74. Roger Guimerà, Marta Sales-Pardo, and Luís A. Nunes Amaral. Module identification in bipartite and directed networks. Physical Review E, 76:036102, September 2007.

75. Pascal Held and Rudolf Kruse. Analysis and Visualization of dynamic clusterings. In Proceedings of the 46th Hawaii International Conference on System Sciences, pages 13851393, 2013. 
76. John E. Hopcroft, Omar Khan, Brian Kulis, and Bart Selman. Tracking Evolving Communities in Large Linked Networks. Proceedings of the National Academy of Science of the United States of America, 101:5244-5253, April 2004.

77. Paul Jaccard. The distribution of flora in the alpine zone. New Phytologist, 11(2):37-50, February 1912.

78. Ravi Kannan, Santosh Vempala, and Adrian Vetta. On Clusterings: Good, Bad, Spectral. Journal of the ACM, 51(3):497-515, May 2004.

79. Keehyung Kim, Robert Ian McKay, and Byung-Ro Moon. Multiobjective evolutionary algorithms for dynamic social network clustering. In Proceedings of the 12th annual conference on Genetic and evolutionary computation, pages 1179-1186. ACM Press, 2010.

80. Min-Soo Kim and Jiawei Han. A particle-and-density based evolutionary clustering method for dynamic networks. In Proceedings of the 35th International Conference on Very Large Databases (VLDB 2009), pages 622-633, 2009.

81. Ravi Kumar, Jasmine Novak, and Andrew S. Tomkins. Structure and evolution of online social networks. In Proceedings of the 12th ACM SIGKDD International Conference on Knowledge Discovery and Data Mining, pages 611-617. ACM Press, 2006.

82. Jian-Huang Lai, Chang-Dong Wang, and Philip Yu. Dynamic Community Detection in Weighted Graph Streams. In Proceedings of the 2013 SIAM International Conference on Data Mining, pages 151-161. SIAM, 2013.

83. Andrea Lancichinetti and Santo Fortunato. Benchmarks for testing community detection algorithms on directed and weighted graphs with overlapping communities. Physical Review E, 80(1):016118, 2009.

84. Andrea Lancichinetti, Santo Fortunato, and János Kertész. Detecting the overlapping and hierarchical community structure of complex networks. New Journal of Physics, 11(033015), 2009.

85. Andrea Lancichinetti, Santo Fortunato, and Filippo Radicchi. Benchmark graphs for testing community detection algorithms. Physical Review E, 78(4):046110, October 2008.

86. Conrad Lee and Pádraig Cunningham. Benchmarking community detection methods on social media data, 2013. preprint, arXiv:1302.0739 [cs.SI].

87. E. A. Leicht and Mark E. J. Newman. Community Structure in Directed Networks. Physical Review Letters, 100(11):118703+, March 2008.

88. Frank Thomson Leighton and Satish Rao. Multicommodity max-flow min-cut theorems and their use in designing approximation algorithms. Journal of the ACM, 46(6):787-832, 1999.

89. Jure Leskovec, Lars Backstrom, Ravi Kumar, and Andrew S. Tomkins. Microscopic evolution of social networks. In Proceedings of the 14th ACM SIGKDD International Conference on Knowledge Discovery and Data Mining, pages 462-470. ACM Press, 2008.

90. Jure Leskovec, Jon M. Kleinberg, and Christos Faloutsos. Graphs Over Time: Densification Laws, Shrinking Diameters and Possible Explanations. In Proceedings of the 11th ACM SIGKDD International Conference on Knowledge Discovery and Data Mining, pages 177187. ACM Press, 2005.

91. Yu-Ru Lin, Yun Chi, Shenghuo Zhu, Hari Sundaram, and Belle L. Tseng. Analyzing communities and their evolutions in dynamic social networks. ACM Transactions on Knowledge Discovery from Data, 3(2):8:1-8:31, April 2009.

92. Feng Luo, James Z. Wang, and Eric Promislow. Exploring Local Community Structures in Large Networks. In IEEE/WIC/ACM International Conference on Web Intelligence, pages 233-239. IEEE, 2006.

93. Henning Meyerhenke. Dynamic Load Balancing for Parallel Numerical Simulations Based on Repartitioning with Disturbed Diffusion. In 15th International Conference on Parallel and Distributed Systems (ICPADS), pages 150-157. IEEE, 2009. 
94. Henning Meyerhenke, Burkhard Monien, and Thomas Sauerwald. A new diffusion-based multilevel algorithm for computing graph partitions. Journal of Parallel and Distributed Computing, 69(9):750-761, 2009.

95. Henning Meyerhenke, Burkhard Monien, and Stefan Schamberger. Graph Partitioning and Disturbed Diffusion. Parallel Computing, 35(10-11):544-569, October 2009.

96. Boris Mirkin. Eleven Ways to Look at the Chi-Squared Coefficient for Contingency Tables. The American Statistician, 55(2):111-120, May 2001.

97. James Moody, Daniel McFarland, and Skye Bender-deMoll. Dynamic Network Visualization. American Journal of Sociology, 110(4):1206-1241, 2005.

98. Chris Muelder and Kwan-Liu Ma. A Treemap Based Method for Rapid Layout of Large Graphs. In Proceedings of IEEE Pacific Visualization Symposium, pages 231-238, 2008.

99. Chris Muelder and Kwan-Liu Ma. Rapid Graph Layout Using Space Filling Curves. IEEE Transactions on Visualization and Computer Graphics, 14(6):1301-1308, 2008.

100. Mark E. J. Newman. The Structure and Function of Complex Networks. SIAM Review, 45(2):167-256, 2003.

101. Mark E. J. Newman. Analysis of Weighted Networks. Physical Review E, 70(056131):1-9, 2004.

102. Mark E. J. Newman. Detecting Community Structure in Networks. The European Physical Journal B, 38(2):321-330, 2004.

103. Mark E. J. Newman and Michelle Girvan. Finding and evaluating community structure in networks. Physical Review E, 69(026113):1-16, 2004.

104. N. P. Nguyen, Thang N. Dinh, Xuan Ying, and My T. Thai. Adaptive algorithms for detecting community structure in dynamic social networks. In Proceedings of the 30th Annual Joint Conference of the IEEE Computer and Communications Societies (Infocom), pages 2282-2290. IEEE Computer Society Press, 2011.

105. Vincenzo Nicosia, G. Mangioni, V. Carchiolo, and M. Malgeri. Extending the Definition of Modularity to Directed Graphs with Overlapping Communities. Journal of Statistical Mechanics: Theory and Experiment, 2009(03):p03024 (23pp), 2009.

106. Huazhong Ning, Wei Xu, Yun Chi, Yihong Gong, and Thomas Huang. Incremental Spectral Clustering With Application to Monitoring of Evolving Blog Communities. In Proceedings of the 2007 SIAM International Conference on Data Mining, pages 261-272. SIAM, 2007.

107. Huazhong Ning, Wei Xu, Yun Chi, Yihong Gong, and Thomas Huang. Incremental spectral clustering by efficiently updating the eigen-system. Pattern Recognition, 43:113-127, 2010.

108. Michael Ovelgönne and Andreas Geyer-Schulz. An ensemble learning strategy for graph clustering. In David A. Bader, Henning Meyerhenke, Peter Sanders, and Dorothea Wagner, editors, Graph Partitioning and Graph Clustering: Tenth DIMACS Implementation Challenge, volume 588 of DIMACS Book, pages 187-206. American Mathematical Society, 2013.

109. Gergely Palla, Albert-László Barabási, and Tamás Vicsek. Quantifying social group evolution. Nature, 446:664-667, April 2007.

110. Gergely Palla, Imre Derényi, Illés Farkas, and Tamás Vicsek. Uncovering the overlapping community structure of complex networks in nature and society. Nature, 435:814-818, 2005.

111. Sheng Pang, Changija Chen, and Ting Wei. A realtime community detection algorithm: incremental label propagation. In First International Conference on Future Information Networks (ICFIN'09), pages 313-317. IEEE, 2009.

112. YoungJa Park and ManSuk Song. A Genetic Algorithm for Clustering Problems. In Proceedings of the 3rd Annual Conference on Genetic Programming, pages 568-575, 1998.

113. Rob Patro, Geet Duggal, Emre Sefer, Hao Wang, Darya Filippova, and Carl Kingsford. The Missing Models: A Data-Driven Approach for Learning How Networks Grow . In 
Proceedings of the 18th ACM SIGKDD International Conference on Knowledge Discovery and Data Mining, pages 42-50. ACM Press, 2012.

114. Karl Pearson. On the criterion that a given system of deviations from the probable in the case of a correlated system of variables is such that it can be reasonably supposed to have arisen from random sampling. Philosophical Magazine Series 5, 50(302):157-175, 1900.

115. Pascal Pons and Matthieu Latapy. Computing Communities in Large Networks Using Random Walks. Journal of Graph Algorithms and Applications, 10(2):191-218, 2006.

116. Usha Nandini Raghavan, Réka Albert, and Soundar Kumara. Near linear time algorithm to detect community structures in large-scale networks. Physical Review E, 76(3):036106, September 2007.

117. William M. Rand. Objective Criteria for the Evaluation of Clustering Methods. Journal of the American Statistical Association, 66(336):846-850, December 1971.

118. Jason Riedy and David A. Bader. Multithreaded Community Monitoring for Massive Streaming Graph Data. In Workshop on Multithreaded Architectures and Applications (MTAAP 2013), 2013. to appear.

119. Jason Riedy, Henning Meyerhenke, David Edinger, and David A. Bader. Parallel Community Detection for Massive Graphs. In Roman Wyrzykowski, Jack Dongarra, Konrad Karczewski, and Jerzy Waśniewski, editors, Parallel Processing and Applied Mathematics, volume 7203 of Lecture Notes in Computer Science, pages 286-296. Springer, 2012.

120. Jorma Rissanen. Modeling by shortest data description. Automatica, 14(5):465-471, September 1978.

121. Randolf Rotta and Andreas Noack. Multilevel local search algorithms for modularity clustering. ACM Journal of Experimental Algorithmics, 16:2.3:2.1-2.3:2.27, July 2011.

122. Peter J. Rousseeuw. Silhouettes: A graphical aid to the interpretation and validation of cluster analysis. Journal of Computational and Applied Mathematics, 20(0):53-65, 1987.

123. Barna Saha and Pabitra Mitra. Dynamic Algorithm for Graph Clustering Using Minimum Cut Tree. In Proceedings of the Sixth IEEE International Conference on Data Mining Workshops, pages 667-671. IEEE Computer Society, December 2006.

124. Barna Saha and Pabitra Mitra. Dynamic Algorithm for Graph Clustering Using Minimum Cut Tree. In Proceedings of the 2007 SIAM International Conference on Data Mining, pages 581-586. SIAM, 2007.

125. Arnaud Sallaberry, Chris Muelder, and Kwan-Liu Ma. Clustering, Visualizing, and Navigating for Large Dynamic Graphs. In Walter Didimo and Maurizio Patrignani, editors, Proceedings of the 20th International Symposium on Graph Drawing (GD'12), volume 7704 of Lecture Notes in Computer Science, pages 487-498. Springer, 2013.

126. E. N. Sawardecker, Marta Sales-Pardo, and Luís A. Nunes Amaral. Detection of node group membership in networks with group overlap. The European Physical Journal B, 67:277-284, 2009.

127. Satu Elisa Schaeffer. Graph Clustering. Computer Science Review, 1(1):27-64, August 2007.

128. Philipp Schuetz and Amedeo Caflisch. Efficient Modularity Optimization by Multistep Greedy Algorithm and Vertex Mover Refinement. Physical Review E, 77(046112), 2008.

129. Huawei Shen, Xueqi Cheng, Kai Cai, and Mao-Bin Hu. Detect overlapping and hierarchical community structure in networks. Physica A: Statistical Mechanics and its Applications, 388(8):1706-1712, 2009.

130. Jianbo Shi and Jitendra Malik. Normalized Cuts and Image Segmentation. IEEE Transactions on Pattern Analysis and Machine Intelligence, 22(8):888-905, 2000.

131. R. Sibson. SLINK: An optimally efficient algorithm for the single-link cluster method. The Computer Journal, 16(1):30-34, January 1973. 
132. Jiř́ Š́ma and Satu Elisa Schaeffer. On the NP-Completeness of Some Graph Cluster Measures. In Proceedings of the 32rd International Conference on Current Trends in Theory and Practice of Computer Science (SOFSEM'06), Lecture Notes in Computer Science, pages 530-537. Springer, 2006.

133. Tom A.B. Snijders and Krzysztof Nowicki. Estimation and Prediction of Stochastic Blockmodels for Graphs with Latent Block Structure. Journal of Classification, 14:75-100, 1997.

134. Myra Spiliopoulou, Irene Ntoutsi, Yannis Theodoridis, and Rene Schult. MONIC: modeling and monitoring cluster transitions. In Proceedings of the 12th ACM SIGKDD International Conference on Knowledge Discovery and Data Mining, pages 706-711. ACM Press, 2006.

135. Isabelle Stanton and Gabriel Kliot. Streaming Graph Partitioning for Large Distributed Graphs. In Proceedings of the 18th ACM SIGKDD International Conference on Knowledge Discovery and Data Mining, pages 1222-1230. ACM Press, 2012.

136. Christian Staudt and Henning Meyerhenke. Engineering High-Performance Community Detection Heuristics for Massive Graphs. In Proceedings of the 2013 International Conference on Parallel Processing. Conference Publishing Services (CPS), 2013.

137. Jimeng Sun, Philip S. Yu, Spiros Papadimitriou, and Christos Faloutsos. GraphScope: Parameter-Free Mining of Large Time-Evolving Graphs. In Proceedings of the 13th ACM SIGKDD International Conference on Knowledge Discovery and Data Mining, pages 687696. ACM Press, 2007.

138. Yizhou Sun, Jie Tang, Jiawei Han, Manish Gupta, and Bo Zhao. Community evolution detection in dynamic heterogeneous information networks. In Proceedings of the Eighth Workshop on Mining and Learning with Graphs, pages 137-146. ACM Press, 2010.

139. Yizhou Sun, Yintao Yu, and Jiawei Han. Ranking-based Clustering of Heterogeneous Information Networks with Star Network Schema. In Proceedings of the 15th ACM SIGKDD International Conference on Knowledge Discovery and Data Mining, pages 797-806. ACM Press, 2009.

140. Siva R. Sundaresan, Ilya R. Fischhoff, and Jonathan Dushoff. Network metrics reveal differences in social organization between two fission-fusion species, Grevy's zebra and onager. Oecologia, 151(1):140-149, 2007.

141. Mansoureh Takaffoli, Justin Fagnan, Farzad Sangi, and Osmar R. Zaïane. Tracking changes in dynamic information networks. In Proceedings of the 2011 IEEE International Conference on Computational Aspects of Social Networks, pages 94-101. IEEE Computer Society, 2011.

142. Mansoureh Takaffoli, Reihaneh Rabbany, and Osmar R. Zaïane. Incremental Local Community Identification in Dynamic Social Networks. In Proceedings of the 2013 IEEE/ACM International Conference on Advances in Social Networks Analysis and Mining. IEEE Computer Society, 2013. to appear.

143. HangHang Tong, Spiros Papadimitriou, Jimeng Sun, Philip S. Yu, and Christos Faloutsos. Colibri: fast mining of large static and dynamic graphs. In Proceedings of the 14th ACM SIGKDD International Conference on Knowledge Discovery and Data Mining, pages 686694. ACM Press, 2008.

144. Alexei Vázquez. Growing network with local rules: Preferential attachment, clustering hierarchy, and degree correlations. Physical Review E, 67:056104, May 2003.

145. Bimal Viswanath, Alan Mislove, Meeyoung Cha, and P. Krishna Gummadi. On the Evolution of User Interaction in Facebook. In Proceedings of the 2Nd ACM Workshop on Online Social Networks, pages 37-42. ACM Press, 2009.

146. Ulrike von Luxburg. A Tutorial on Spectral Clustering. Statistics and Computing, 17(4):395-416, December 2007.

147. Silke Wagner and Dorothea Wagner. Comparing Clusterings - An Overview. Technical Report 2006-04, iti_wagner, 2007. 
148. Yuchung J. Wang and George Y. Wong. Stochastic Blockmodels for Directed Graphs. Journal of the American Statistical Association, 82:8-19, 1987.

149. Duncan J. Watts. Networks, Dynamics, and the Small-World Phenomenon. American Journal of Sociology, 105:493-527, 1999.

150. Duncan J. Watts and Steven H. Strogatz. Collective dynamics of 'small-world' networks. Nature, 393(6684):440-442, June 1998.

151. Jierui Xie, Mingming Chen, and Boleslaw K. Szymanski. LabelRankT: Incremental Community Detection in Dynamic Networks via Label Propagation. CoRR, abs/1305.2006, 2013.

152. Jierui Xie and Boleslaw K. Szymanski. LabelRank: A Stabilized Label Propagation Algorithm for Community Detection in Networks. CoRR, abs/1303.0868, 2013.

153. Kevin S. Xu, Mark Kliger, and Alfred O. Hero III. Tracking Communities in Dynamic Social Networks. In Proceedings of the 4th International Conference on Social Computing, Behavioral-Cultural Modeling and Prediction, pages 219-226, 2011.

154. Xiaowei Xu, Nurcan Yuruk, Zhidan Feng, and Thomas A. J. Schweiger. SCAN: A Structural Clustering Algorithm for Networks. In Proceedings of the 13th ACM SIGKDD International Conference on Knowledge Discovery and Data Mining, pages 824-833. ACM Press, 2007.

155. Tianbao Yang, Yun Chi, Shenghuo Zhu, and Rong Jin. Detecting communities and their evolutions in dynamic social networks - a Bayesian approach. Machine Learning, 82(2):157-189, February 2011.

156. Kai Yu, Shipeng Yu, and Volker Tresp. Soft Clustering on Graphs. In Advances in Neural Information Processing Systems 18, page 05. MIT Press, 2006.

157. Stella X. Yu and Jianbo Shi. Multiclass spectral clustering. In Proceedings of the 9th IEEE International Conference on Computer Vision, pages 313-319, 2003.

158. Wayne W. Zachary. An Information Flow Model for Conflict and Fission in Small Groups. Journal of Anthropological Research, 33:452-473, 1977.

159. Yuchen Zhao and Philip S. Yu. On Graph Stream Clustering with Side Information. In Proceedings of the seventh SIAM International Conference on Data Mining, pages 139150. SIAM, 2013.

160. Elena Zheleva, Hossam Sharara, and Lise Getoor. Co-evolution of social and affiliation networks. In Proceedings of the 15th ACM SIGKDD International Conference on Knowledge Discovery and Data Mining, pages 1007-1016. ACM Press, 2009.

161. Haijun Zhou. Network landscape from a Brownian particle's perspective. Physical Review E, 67:041908, 2003. 